\title{
Exotic electronic and transport properties of graphene
}

\author{
Tsuneya Ando ${ }^{1}$ \\ Department of Physics, Tokyo Institute of Technology \\ 2-12-1 Ookayama, Meguro-ku, Tokyo, 152-8551, Japan.
}

\begin{abstract}
A brief review is given on electronic and transport properties of monolayer graphene from a theoretical point of view. The topics include the effective-mass description of electronic states, topological anomaly associated with Berry's phase, singular diamagnetic susceptibility, zero-mode anomalies and their removal due to level broadening effects, screening effect and charged impurity scattering, the symmetry crossover among symplectic, unitary, and orthogonal due to the presence of special time reversal symmetry, and anomaly and magnetic oscillation of long-wavelength optical phonons.
\end{abstract}

Key words: honeycomb lattice, neutrino, topological anomaly, quantum Hall effect, zero-mode anomaly, screening, charged impurity scattering, optical phonon, magneto-phonon resonance

PACS: 78.67.Ch, 73.22.Lp

\section{Introduction}

In an effective-mass approximation, an electron in a graphite monolayer or graphene is described by Weyl's equation for a massless neutrino [1-4]. Transport properties in such an exotic electronic structure are quite intriguing, and the conductivity with/without a magnetic field including the Hall effect $[5,6]$, quantum corrections to the conductivity [7], and the dynamical transport [8] were investigated theoretically. The results show that the system exhibits various characteristic behaviors different from conventional twodimensional systems [9]. Quite recently, this single layer graphene was fabricated [10] and the magnetotransport was measured including the integer quantum Hall effect, demonstrating the validity of the neutrino

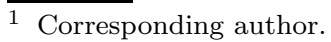

E-mail: ando@phys.titech.ac.jp
}

description of the electronic states [11,12]. Since then, the graphene became the subject of extensive theoretical [13-21] and experimental study [22-24]. The purpose of this paper is to give a brief review on such electronic and transport properties from a theoretical point of view.

The effective-mass description of electronic states is discussed in Sec. 2. The topological anomaly associated with Berry's phase and the resulting unique Landaulevel structure are discussed in Sec. 3. The singular diamagnetic susceptibility is reviewed in Sec. 4. In Sec. 5 , various zero-mode anomalies appearing in transport quantities and their removal due to level broadening effects are discussed. The screening effect and impurity scattering are reviewed briefly in Sec. 6. The presence of special time-reversal symmetry and associated symmetry crossover are discussed in Sec. 7. An anomaly appearing in long-wavelength optical phonons and magnetic oscillation are discussed in Sec. 8. 


\section{Neutrino Description}

The structure of 2D graphite sheet is shown in Fig. 1. A unit cell contains two carbon atoms which are denoted by $\mathrm{A}$ and $\mathrm{B}$. We have the primitive translation vectors $\mathbf{a}=a(1,0)$ and $\mathbf{b}=a(-1 / 2, \sqrt{3} / 2)$, and the vectors connecting between nearest neighbor carbon atoms $\boldsymbol{\tau}_{1}=a(0,1 / \sqrt{3}), \boldsymbol{\tau}_{2}=a(-1 / 2,-1 / 2 \sqrt{3})$, and $\boldsymbol{\tau}_{3}=a(1 / 2,-1 / 2 \sqrt{3})$, where the lattice constant is given by $a=2.46 \AA$. The first Brillouin zone is given by a hexagon with two corner points $\mathrm{K}$ and $\mathrm{K}$ '. The corresponding wave vectors are given by $\mathbf{K}=$ $(2 \pi / a)(1 / 3,1 / \sqrt{3})$ and $\mathbf{K}^{\prime}=(2 \pi / a)(2 / 3,0)$ for $\mathbf{K}$ and $\mathrm{K}^{\prime}$ points, respectively. In a nearest-neighbor tightbinding model, the energy bands are given by

$$
\varepsilon_{ \pm}(\mathbf{k})= \pm \gamma_{0}\left|\sum_{l=1}^{3} \exp \left(i \mathbf{k} \cdot \boldsymbol{\tau}_{l}\right)\right|
$$

The band structure is shown in Fig. 2. Near the K and $\mathrm{K}^{\prime}$ point, we have $\varepsilon_{ \pm}(\mathbf{k}+\mathbf{K})=\varepsilon_{ \pm}\left(\mathbf{k}+\mathbf{K}^{\prime}\right)= \pm \gamma|\mathbf{k}|$ with $\gamma=\sqrt{3} a \gamma_{0} / 2$.

Essential and important features of electronic states become transparent when we use a $\mathbf{k} \cdot \mathbf{p}$ scheme in describing states in the vicinity of $\mathrm{K}$ and $\mathrm{K}$ ' points. The effective-mass equation for the $\mathrm{K}$ point is given by

$$
\gamma(\boldsymbol{\sigma} \cdot \hat{\mathbf{k}}) \mathbf{F}(\mathbf{r})=\varepsilon \mathbf{F}(\mathbf{r}), \quad \mathbf{F}(\mathbf{r})=\left(\begin{array}{c}
F_{A}(\mathbf{r}) \\
F_{B}(\mathbf{r})
\end{array}\right),
$$

where $\boldsymbol{\sigma}=\left(\sigma_{x}, \sigma_{y}\right)$ is the Pauli spin matrix, $\gamma$ is a band parameter, $\hat{\mathbf{k}}=-i \boldsymbol{\nabla}$, and $F_{A}$ and $F_{B}$ represent the amplitude at two carbon sites $\mathrm{A}$ and $\mathrm{B}$, respectively [25]. The above equation is same as Weyl's equation for a neutrino with vanishing rest mass and constant
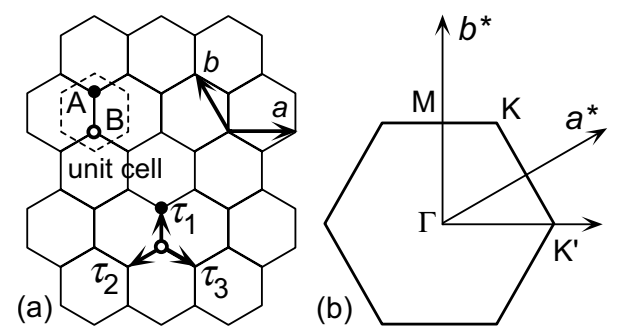

Fig. 1. The lattice structure (a) and the first Brillouin zone (b) of graphene. The unit cell contains two carbon atoms denoted by A and B. Two primitive translation vectors are denoted by $\mathbf{a}$ and $\mathbf{b}$ and three vectors connecting neighboring $\mathrm{A}$ and $\mathrm{B}$ atoms are $\boldsymbol{\tau}_{l}(l=1,2,3)$. Reciprocal lattice vectors are given by $\mathbf{a}^{*}$ and $\mathbf{b}^{*}$.

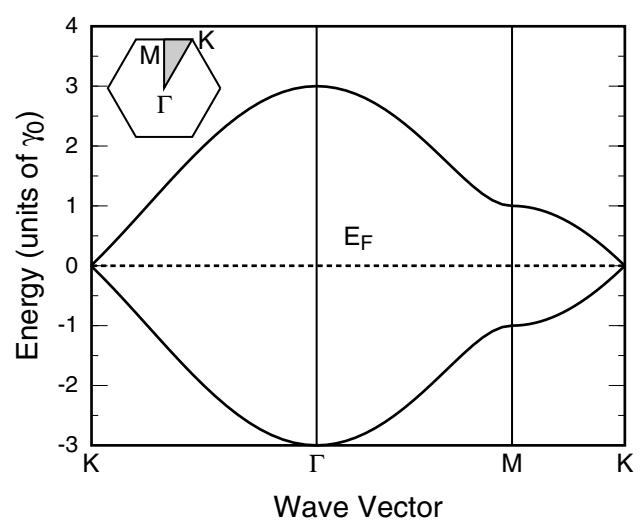

Fig. 2. The band structure obtained in a tight-binding model.

velocity independent of the wave vector. The velocity is given by $|\mathbf{v}|=\gamma / \hbar$.

The wave function is written as

$$
\begin{aligned}
& \mathbf{F}_{s \mathbf{k}}(\mathbf{r})=\frac{1}{L} \mathbf{F}_{s \mathbf{k}} \exp (i \mathbf{k} \cdot \mathbf{r}), \\
& \mathbf{F}_{s \mathbf{k}}=\frac{1}{\sqrt{2}}\left(\begin{array}{c}
e^{-i \theta_{\mathbf{k}}} \\
s
\end{array}\right)
\end{aligned}
$$

where $L^{2}$ is the area of the system, $s=+1$ and -1 denote the conduction and valence bands, respectively, and

$$
k_{x}=k \cos \theta_{\mathbf{k}}, \quad k_{y}=k \sin \theta_{\mathbf{k}}, \quad k=\sqrt{k_{x}^{2}+k_{y}^{2}} .
$$

The corresponding energy is given by

$$
\varepsilon_{s \mathbf{k}}=s \varepsilon_{\mathbf{k}}, \quad \varepsilon_{\mathbf{k}}=\gamma|\mathbf{k}| .
$$

For the K' point the Schrödinger equation is given by Eq. (2) in which $\boldsymbol{\sigma}$ is replaced by its complex conjugate $\boldsymbol{\sigma}^{*}$. The density of states becomes $D(\varepsilon)=$ $g_{\mathrm{v}} g_{\mathrm{s}}|\varepsilon| / 2 \pi \gamma^{2}$, with the spin $g_{\mathrm{v}}=2$ and valley degeneracy $g_{\mathrm{v}}=2$. Figure 3 shows the energy dispersion and the density of states.

\section{Topological Anomaly and Berry's Phase}

An important feature is the presence of a topological singularity at $\mathbf{k}=0$. A neutrino has a helicity and its spin is quantized into the direction of its motion. The spin eigen function changes its sign under a $2 \pi$ rotation. For the present pseudo spin, this sign change can be understood in terms of Berry's phase $e^{-i \eta}$, given by 

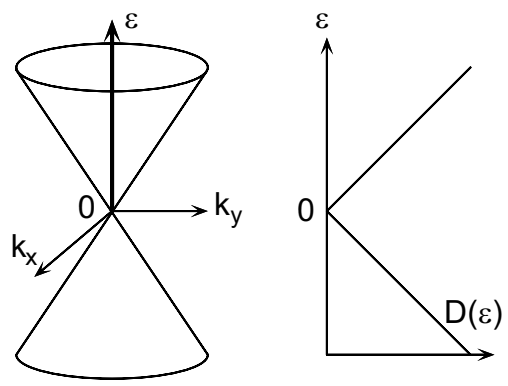

Fig. 3. The energy dispersion and density of states in the vicinity of $K$ and $K^{\prime}$ points obtained in a $\mathbf{k} \cdot \mathbf{p}$ scheme.

$$
\eta=-i \int_{0}^{T} d t\left\langle s \mathbf{k}(t)\left|\frac{d}{d t}\right| s \mathbf{k}(t)\right\rangle=-\pi,
$$

where $\mathbf{k}(t)$ moves along a closed contour around $\mathbf{k}=0$ between time $t=0$ and $T$. Therefore the wave function acquires phase $-\pi$ when $\mathbf{k}$ is rotated around the origin [4,26-28]. The signature change occurs only when the closed contour encircles the origin $\mathbf{k}=0$ but not when the contour does not contain $\mathbf{k}=0$. This topological singularity at $\mathbf{k}=0$ and associated Berry's phase are the origin of the absence of backward scattering in metallic carbon nanotubes $[4,26,27]$.

A singularity at $\varepsilon=0$ manifests itself in magnetic fields even in classical mechanics. The equation of motion gives the cyclotron frequency $\omega_{c}=e B v^{2} / c \varepsilon$. The cyclotron frequency $\omega_{c}$ diverges and changes its signature at $\varepsilon=0[6,16]$.

In quantum mechanics $\hat{k}_{x}$ and $\hat{k}_{y}$ satisfy the commutation relation $\left[\hat{k}_{x}, \hat{k}_{y}\right]=-i / l^{2}$, where $l$ is the magnetic length given by $l=\sqrt{c \hbar / e B}$. Semiclassically, the Landau levels can be obtained by the condition

$$
\oint k_{x} d k_{y}= \pm \frac{2 \pi}{l^{2}}\left(|n|+\frac{1}{2}\right)
$$

as $\varepsilon_{n}=\operatorname{sgn}(n) \sqrt{|n|+1 / 2} \hbar \omega_{B}$ with integer $n$, where $\hbar \omega_{B}=\sqrt{2} \gamma / l$ and $\operatorname{sgn}(n)=n /|n|$ for $n \neq 0$ and 0 for $n=0$. Because of the uncertainty principle, $k^{2}=0$ is not allowed and there is no Landau level at $\varepsilon=0$. However, a full quantum mechanical treatment gives $\varepsilon_{n}=\operatorname{sgn}(n) \sqrt{|n|} \hbar \omega_{B}$, leading to the formation of Landau levels at $\varepsilon=0[1]$. This can be understood by the cancellation of factor $1 / 2$ in Eq. (8) by Berry's phase corresponding to the rotation in the $\mathbf{k}$ space.

\section{Singular Diamagnetism}

The unique Landau-level structure gives rise to a singular diamagnetism [1], which is the origin of large diamagnetic susceptibility of bulk graphite $[29,30]$. The thermodynamic function $\Omega$ is given by

$$
\begin{aligned}
& \Omega=-k_{\mathrm{B}} T g_{\mathrm{v}} g_{\mathrm{s}} \frac{1}{2 \pi l^{2}} \sum_{n} \varphi\left(\varepsilon_{n}\right), \\
& \varphi(\varepsilon)=\ln \{1+\exp [\beta(\zeta-\varepsilon)]\},
\end{aligned}
$$

where $T$ is the temperature, $k_{\mathrm{B}}$ is the Boltzmann constant, $\beta=1 / k_{\mathrm{B}} T$, and $\zeta$ the chemical potential. Let $n_{\mathrm{s}}$ be the electron concentration. Then, the free energy is written as

$$
F=n_{\mathrm{s}} \zeta+\Omega .
$$

With the use of the relation $n_{\mathrm{s}}=-(\partial \Omega / \partial \zeta)_{B}$, the magnetization is given by

$$
M=-\left(\frac{\partial F}{\partial B}\right)_{n_{\mathrm{s}}}=-\left(\frac{\partial \Omega}{\partial B}\right)_{\zeta} .
$$

Therefore, susceptibility $\chi$, defined by $M=\chi B$ for small $B$, can be obtained by calculating $\Omega$ up to the order of $B^{2}$.

The thermodynamic function is rewritten as

$$
\begin{aligned}
& \Omega=-k_{\mathrm{B}} T g_{\mathrm{v}} g_{\mathrm{s}} \frac{1}{2 \pi l^{2}} \sum_{n=0}^{\infty} g\left(\hbar \omega_{B} \sqrt{n}\right)\left(1-\frac{1}{2} \delta_{n 0}\right) \\
& \times \ln \left[1+2 \exp (\beta \zeta) \cosh \left(\beta \hbar \omega_{B} \sqrt{n}\right)+\exp (2 \beta \zeta)\right]
\end{aligned}
$$

where $1 / 2 \pi l^{2}$ is the degeneracy of a Landau level and $g(\varepsilon)$ is a cutoff function which decays smoothly but sufficiently rapidly in such a way that the summation converges. For example, we can choose

$$
g(\varepsilon)=\frac{\varepsilon_{c}^{\alpha_{c}}}{\varepsilon^{\alpha_{c}}+\varepsilon_{c}^{\alpha_{c}}}
$$

with cutoff energy $\varepsilon_{c}$ and parameter $\alpha_{c}\left(\alpha_{c} \geq 2\right)$.

Consider a smooth function $F(x)$ and its integral

$$
\int_{0}^{\infty} F(x) d x=\int_{0}^{h / 2} F(x) d x+\sum_{j=1}^{\infty} \int_{-h / 2}^{h / 2} F(x+h j) d x,
$$

where $h$ is a small positive number. By expanding this with respect to $h$, we have

$$
\begin{aligned}
& h\left[\frac{1}{2} F(0)+\sum_{j=1}^{\infty} F(x+h j)\right] \\
= & \int_{0}^{\infty} F(x) d x-\frac{1}{12} h^{2}\left[F^{\prime}(0)+\frac{1}{2} F^{\prime}(\infty)\right],
\end{aligned}
$$


up to the second order in $h$. Let $h=\left(\hbar \omega_{B}\right)^{2}, x=n h$, and

$F(x)=g(\sqrt{x}) \ln [1+2 \exp (\beta \zeta) \cosh (\beta \sqrt{x})+\exp (2 \beta \zeta)]$.

Then, we have

$$
\Omega=\Omega_{0}+\Delta \Omega
$$

where $\Omega_{0}$ is the thermodynamic potential in the absence of a magnetic field and

$$
\begin{aligned}
\Delta \Omega & =\frac{1}{12} \frac{g_{\mathrm{v}} g_{\mathrm{s}}\left(\hbar \omega_{B}\right)^{2}}{2 \pi l^{2}} \frac{\beta \exp (\beta \zeta)}{[1+\exp (\beta \zeta)]^{2}} \\
& =\frac{g_{\mathrm{v}} g_{\mathrm{s}} \gamma^{2}}{12 \pi l^{4}} \int_{-\infty}^{\infty}\left(-\frac{\partial f(\varepsilon)}{\partial \varepsilon}\right) \delta(\varepsilon) d \varepsilon .
\end{aligned}
$$

The susceptibility becomes

$$
\chi=-\frac{g_{\mathrm{v}} g_{\mathrm{s}} \gamma^{2}}{6 \pi}\left(\frac{e}{c \hbar}\right)^{2} \int_{-\infty}^{\infty}\left(-\frac{\partial f(\varepsilon)}{\partial \varepsilon}\right) \delta(\varepsilon) d \varepsilon .
$$

This singular susceptibility is characteristic to graphene and obtained first by McClure [1] and later by many others [31-33].

Consider the average contribution of states around $\varepsilon_{n}(|n| \gg 1)$ to the thermodynamic potential in the weak-field limit. We have

$$
\begin{aligned}
& \int_{-1 / 2}^{1 / 2} \operatorname{sgn}(n) \hbar \omega_{B} \sqrt{|n|+t} \varphi\left[\operatorname{sgn}(n) \hbar \omega_{B} \sqrt{|n|+t}\right] d t \\
= & \varphi\left(\varepsilon_{n}\right)+\frac{\beta}{96}\left(\hbar \omega_{B}\right)^{4}\left[\varepsilon_{n}^{-3} f\left(\varepsilon_{n}\right)-\varepsilon_{n}^{-2} f^{\prime}\left(\varepsilon_{n}\right)\right]+\cdots .(21)
\end{aligned}
$$

Therefore, the change in the thermodynamic potential in a magnetic field becomes

$$
\begin{aligned}
\Delta \Omega & =\frac{g_{\mathrm{v}} g_{\mathrm{s}}}{2 \pi l^{2}} \frac{1}{96}\left(\hbar \omega_{B}\right)^{4} \sum_{n}\left[\varepsilon_{n}^{-3} f\left(\varepsilon_{n}\right)-\varepsilon_{n}^{-2} f^{\prime}\left(\varepsilon_{n}\right)\right] \\
& =\frac{g_{\mathrm{v}} g_{\mathrm{s}}}{2 \pi l^{2}} \frac{\left(\hbar \omega_{B}\right)^{2}}{48} \int_{-\infty}^{\infty} \operatorname{sgn}(\varepsilon)\left[\varepsilon^{-2} f(\varepsilon)-\varepsilon^{-1} f^{\prime}(\varepsilon)\right] d \varepsilon .
\end{aligned}
$$

When the Fermi level lies well away from $\varepsilon=0$, the integral vanishes identically and therefore the susceptibility vanishes.

At $\varepsilon=0$, the integrand becomes singular and gives the "paramagnetic" contribution

$$
\Delta \Omega=-\frac{g_{\mathrm{v}} g_{\mathrm{s}}}{2 \pi l^{2}} \frac{1}{24}\left(\hbar \omega_{B}\right)^{2} \int_{-\infty}^{\infty}\left(-\frac{\partial f(\varepsilon)}{\partial \varepsilon}\right) \delta(\varepsilon) d \varepsilon .
$$

In fact, we have

$$
\begin{aligned}
& \int_{0}^{\infty}\left(\frac{f(\varepsilon)}{\varepsilon^{2}}-\frac{f^{\prime}(\varepsilon)}{\varepsilon}\right) d \varepsilon-\int_{-\infty}^{0}\left(\frac{f(\varepsilon)}{\varepsilon^{2}}-\frac{f^{\prime}(\varepsilon)}{\varepsilon}\right) d \varepsilon \\
= & -\left.\frac{f(\varepsilon)}{\varepsilon}\right|_{+0} ^{\infty}+\left.\frac{f(\varepsilon)}{\varepsilon}\right|_{-\infty} ^{-0}=\lim _{\varepsilon \rightarrow+0}\left(\frac{f(\varepsilon)}{\varepsilon}+\frac{f(-\varepsilon)}{-\varepsilon}\right) \\
= & 2 f^{\prime}(0) .
\end{aligned}
$$

For $n=0$, the average contribution to the thermodynamic potential in the weak-field limit becomes

$$
\begin{aligned}
\int_{-1 / 2}^{1 / 2} \varphi\left[\operatorname{sgn}(t) \hbar \omega_{B} \sqrt{|t|}\right] d t & =\frac{1}{8}\left(\hbar \omega_{B}\right)^{2} \varphi^{\prime \prime}(0)+\cdots \\
& =-\frac{\beta}{8}\left(\hbar \omega_{B}\right)^{2} f^{\prime}(0)+\cdots,
\end{aligned}
$$

giving

$$
\Delta \Omega=\frac{g_{\mathrm{v}} g_{\mathrm{s}}}{2 \pi l^{2}} \frac{1}{8}\left(\hbar \omega_{B}\right)^{2} \int_{-\infty}^{\infty}\left(-\frac{\partial f(\varepsilon)}{\partial \varepsilon}\right) \delta(\varepsilon) d \varepsilon .
$$

By adding the contribution from $n \neq 0$ given by Eq. (23), we have the result given by Eq. (19). This shows clearly that the presence of the Landau level at $\varepsilon=0$ is the origin of the singular behavior of the susceptibility.

In a bilayer graphene the effective Hamiltonian is rewritten as [34,35]

$$
H=\frac{\hbar^{2}}{2 m^{*}}\left(\begin{array}{cc}
0 & \left(\hat{k}_{x}+i \hat{k}_{y}\right)^{2} \\
\left(\hat{k}_{x}-i \hat{k}_{y}\right)^{2} & 0
\end{array}\right) .
$$

This gives the Landau level $\varepsilon_{ \pm n}= \pm \hbar \omega_{\mathrm{c}} \sqrt{n(n+1)}$ with $n=0,1, \cdots$ with $\omega_{c}=e B / m^{*} c$. In a similar but more complicated manner, the susceptibility can be calculated as

$$
\chi=-\left(\frac{e \hbar}{2 m^{*} c}\right)^{2} \frac{g_{\mathrm{v}} g_{\mathrm{s}} m^{*}}{2 \pi \hbar^{2}} \int_{-\infty}^{\infty} g(\varepsilon) \ln \frac{\varepsilon_{c}}{|\varepsilon|}\left(-\frac{\partial f}{\partial \varepsilon}\right) d \varepsilon,
$$

except for a constant term independent of energy. The above agrees with the result obtained previously for a model of intercalated graphite $[33,36]$. The energyindependent term is likely to be dependent on the cutoff function.

\section{Zero Mode Anomalies}

We consider, for example, a system with scatterers with a potential range much smaller than the typical electron wavelength (which is actually infinite at $\varepsilon=$ 0 ) but larger than the lattice constant of graphene [26]. The relaxation time in the absence of a magnetic field 

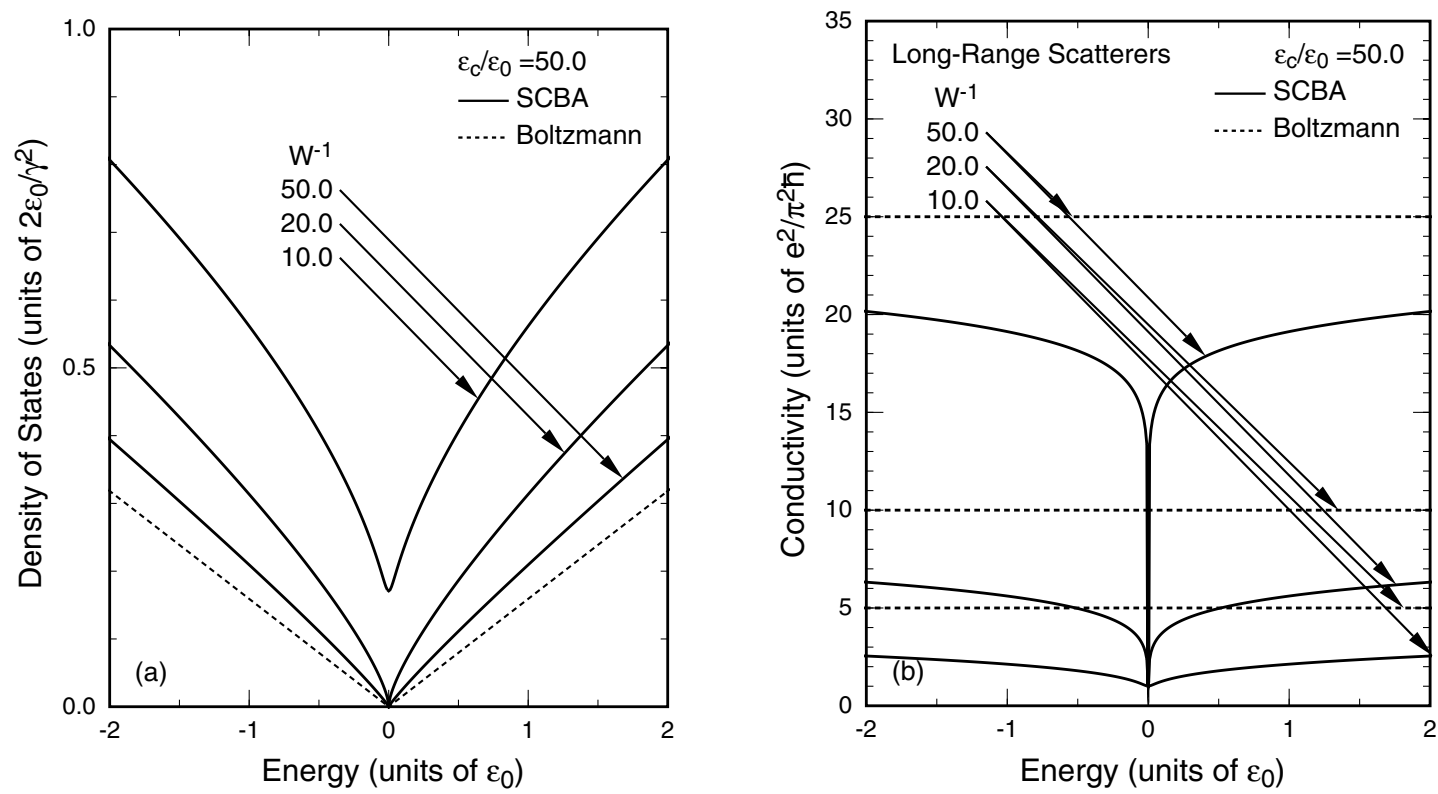

Fig. 4. Some examples of the density of states (a) and the conductivity (b) of a monolayer graphene calculated in the self-consistent Born approximation. $\varepsilon_{0}$ is an arbitrary energy unit and $\varepsilon_{c}$ is the cutoff energy corresponding to the half of the $\pi$-band width. The conductivity exhibits a sharp jump in the limit of weak scattering $(W \ll 1)$ from the Boltzmann result $\sigma_{0}$ for $\varepsilon \neq 0$ down to $\sigma=e^{2} / \pi^{2} \hbar$ at $\varepsilon_{\mathrm{F}}=0$.

becomes $\tau_{0}^{-1}=2 \pi|\varepsilon| W / \hbar$ with $W$ being a dimensionless parameter characterizing the scattering strength given by

$$
W=\frac{\left\langle n_{\mathrm{i}} u_{\mathrm{i}}^{2}\right\rangle}{\pi \gamma^{2}},
$$

where $u_{\mathrm{i}}$ and $n_{\mathrm{i}}$ are the strength and the concentration of scatterers, respectively, and $\langle\cdots\rangle$ means the average over impurities. We usually have $W \ll 1$. With the use of the Boltzmann transport equation, the transport relaxation time becomes $\tau(\varepsilon)=2 \tau_{0}(\varepsilon)$ and the conductivity

$$
\sigma_{0}=g_{\mathrm{v}} g_{\mathrm{s}} \frac{e^{2}}{4 \pi^{2} \hbar} \frac{1}{W},
$$

independent of the Fermi level [5]. This shows that graphene should be regarded as a metal rather than a zero-gap semiconductor. It is interesting to note that the conductivity is nonzero even at $\varepsilon=0$ (when the limit $|\varepsilon| \rightarrow 0$ is taken). However, there can be singularity at $\varepsilon=0$ because the density of states vanishes and strictly speaking the above discussion is not applicable.

In the presence of a magnetic field, the conductivity tensor $\sigma_{\mu \nu}$ with $\mu=x, y$ and $\nu=x, y$ is given by $\sigma_{x x}=$ $\sigma_{y y}=\sigma_{0} /\left[1+\left(\omega_{c} \tau\right)^{2}\right]$ and $\sigma_{x y}=-\sigma_{y x}=-\sigma_{0} \omega_{c} \tau /[1+$ $\left.\left(\omega_{c} \tau\right)^{2}\right]$. Using the explicit expressions for $\omega_{c}$ and $\tau$, we have $\sigma_{x x}=\sigma_{0} \xi^{4} /\left(1+\xi^{4}\right)$ and $\sigma_{x y}=-\sigma_{0} \xi^{2} /(1+$ $\left.\xi^{4}\right)$, with $\xi=\sqrt{2 \pi W}\left(\varepsilon_{\mathrm{F}} / \hbar \omega_{B}\right)$. Because $\tau\left(\varepsilon_{\mathrm{F}}\right)^{-1} \propto$ $\left|\varepsilon_{\mathrm{F}}\right|$, the dependence on the Fermi energy $\varepsilon_{\mathrm{F}}$ is fully scaled by $\hbar \omega_{B}$. Therefore, the conductivity $\sigma_{x x}$ exhibits a singular jump to zero at $\varepsilon_{\mathrm{F}}=0$ from $\sigma_{0}$ for nonzero $\varepsilon_{\mathrm{F}}$ in the limit of the vanishing magnetic field $\hbar \omega_{B} \rightarrow 0$.

A singular behavior appears also in the dynamical conductivity [8]. In a relaxation-time approximation, the frequency dependence of the dynamical conductivity is scaled by the Fermi energy, i.e., $\sigma\left(\omega, \varepsilon_{\mathrm{F}}\right)=$ $\sigma\left(\hbar \omega / \varepsilon_{\mathrm{F}}\right)$. This scaling shows that $\sigma\left(\omega, \varepsilon_{\mathrm{F}}\right)$ exhibits a singular behavior at the point $\left(\omega, \varepsilon_{\mathrm{F}}\right)=(0,0)$. The correct way may be to let $\omega \rightarrow 0$ at each $\varepsilon_{\mathrm{F}}$, leading to a singular jump of the static conductivity to $g_{\mathrm{v}} g_{\mathrm{s}} e^{2} / 16 \hbar$ at $\varepsilon_{\mathrm{F}}=0$ from $\sigma_{0}$ for nonzero $\varepsilon_{\mathrm{F}}$.

A more refined treatment has been performed for the density of states and the conductivity in a self-consistent Born approximation in which levelbroadening effects are properly taken into account [5]. Figure 4 shows an example. The density of states becomes nonzero at $\varepsilon=0$ because of level broadening and also enhanced due to level repulsion effect near $\varepsilon=0$. 

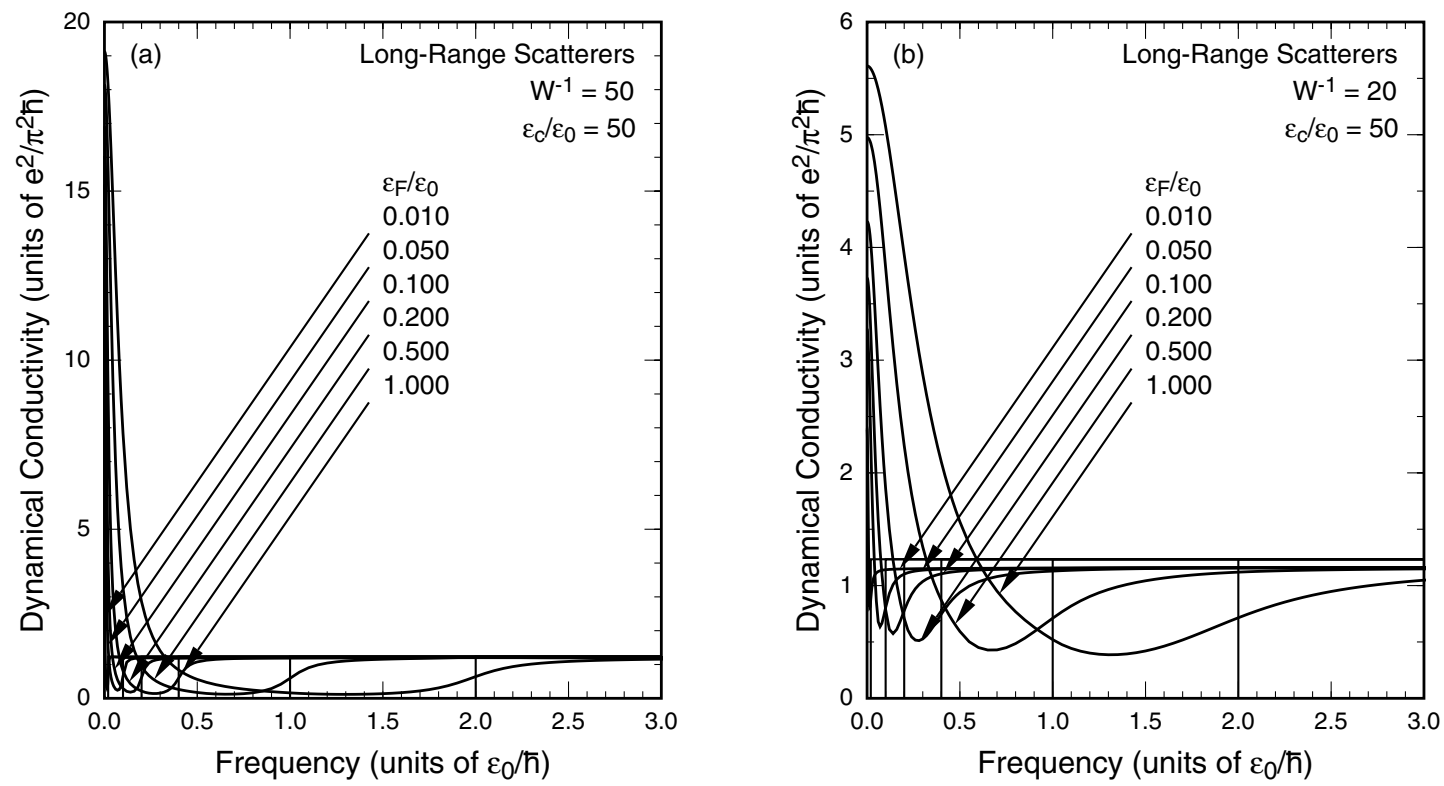

Fig. 5. Some examples of the dynamical conductivity of a monolayer graphene calculated in the self-consistent Born approximation. (a) $W^{-1}=50$. (b) $W^{-1}=20$. The frequency dependence is nearly scaled by $\varepsilon_{\mathrm{F}}$ as long as $\varepsilon_{\mathrm{F}} \neq 0$. This scaling becomes less valid when $\varepsilon_{\mathrm{F}}$ is close to 0 as is more clear in (b).

Further, the conductivity at $\varepsilon_{\mathrm{F}}=0$ is given by $g_{\mathrm{v}} g_{\mathrm{s}} e^{2} / 2 \pi^{2} \hbar$, which is universal and independent of the scattering strength. The resulting conductivity varies smoothly across $\varepsilon_{\mathrm{F}}=0$ but exhibits a sharp jump in the limit of weak scattering $(W \ll 1)$ from the Boltzmann result $\sigma_{0}$ for $\varepsilon \neq 0$ down to $\sigma=g_{\mathrm{v}} g_{\mathrm{s}} e^{2} / \pi^{2} \hbar$ at $\varepsilon_{\mathrm{F}}=0$. A similar calculation for a bilayer graphene shows much smoother variation with energy with minimum value $\sigma \approx g_{\mathrm{v}} g_{\mathrm{s}} e^{2} / 2 \pi^{2} \hbar$ slightly dependent on the scattering strength [35].

The scattering probability $\hbar / \tau\left(\varepsilon_{\mathrm{F}}\right)$ is proportional to the final-state density of states with a coefficient independent of $\varepsilon_{\mathrm{F}}$. Because the density of states is proportional to $\varepsilon_{\mathrm{F}}$, the relaxation time is inversely proportional to $\varepsilon_{\mathrm{F}}$. As a result the mobility is inversely proportional to $n_{\mathrm{s}} \propto \varepsilon_{\mathrm{F}}^{2}$, leading to the conductivity independent of the Fermi energy and the electron concentration. In the case of charged-impurity scattering discussed in the next section, the matrix element itself is proportional to the inverse of the Fermi energy both in the presence and absence of screening. Consequently, the low-temperature mobility becomes independent of the electron concentration and the conductivity increases in proportion to the electron or hole concentration $n_{\mathrm{s}}[16]$.

Calculations in the self-consistent Born approximation were performed also for the dynamical conductivity [8]. Figure 5 shows some examples of the results. The frequency dependence is scaled completely by $\varepsilon_{\mathrm{F}}$ as long as $\varepsilon_{\mathrm{F}} \neq 0$. When $\varepsilon_{\mathrm{F}}$ is very close to 0 , however, the conductivity at $\omega=0$ becomes small and the discrete jump present in the Boltzmann conductivity is removed. The energy scale causing this crossover behavior becomes exponentially small for weaker $W$, i.e., $\propto \exp \left(-W^{-1} / 2\right)$, leading to a singular behavior of the dynamical conductivity in the weak scattering limit.

Figure 6 shows the density of states, the diagonal conductivity $\sigma_{x x}$ [5], and the Hall conductivity $\sigma_{x y}[6]$ in a magnetic field, calculated in the selfconsistent Born approximation. In high magnetic fields, the broadening of the Landau level $n$ becomes $\hbar \omega_{B} \sqrt{2 W\left(1+\delta_{n 0}\right)}$ and the peak value of $\sigma_{x x}$ becomes $\left(|n|+\delta_{n 0}\right)\left(g_{\mathrm{v}} g_{\mathrm{s}} e^{2} / 2 \pi^{2} \hbar\right)$. It is interesting that the conductivity at $\varepsilon=0$ remains $g_{\mathrm{v}} g_{\mathrm{s}} e^{2} / 2 \pi^{2} \hbar$ independent of the magnetic field. Further, the Hall conductivity in the gap between adjacent Landau levels is quantized into $4(j+1 / 2)\left(e^{2} / h\right)$ with integer $j$. Localization effects are not included in the self-consistent Born 

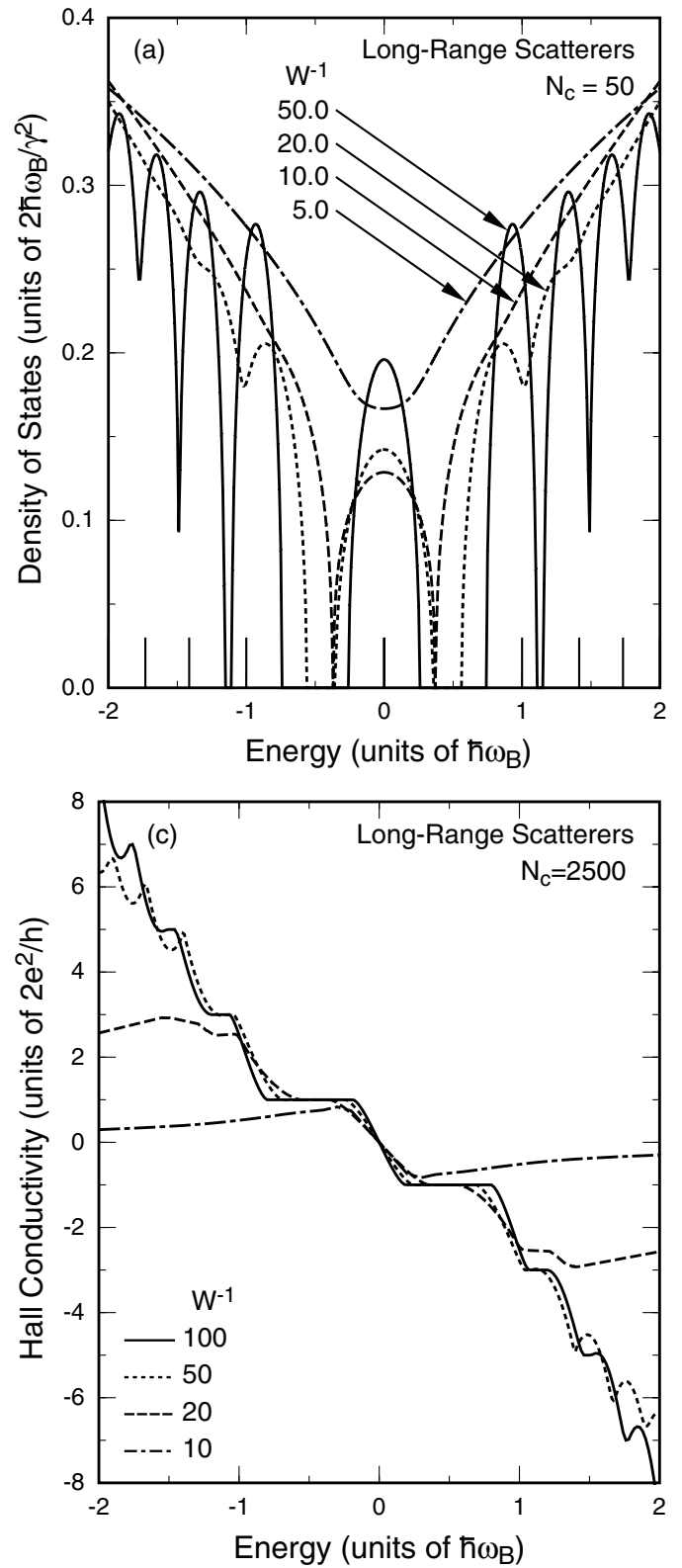

approximation, but it is clear that the Hall conductivity is quantized into these integers when states are localized in sufficiently high magnetic fields [21].

These quantized values are in agreement with the recent experimental observation [11,12]. However, the observed minimum conductivity at zero energy in the absence of a magnetic field seems to be $3 \sim 4$ times as large as the predicted conductivity in the

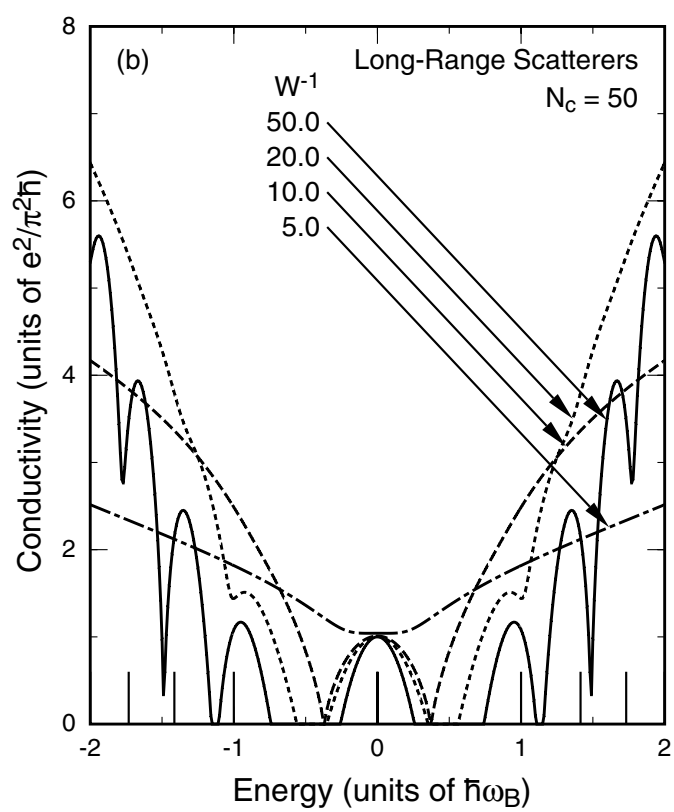

Fig. 6. (a) Some examples of the density of states (a), the diagonal conductivity $\sigma_{x x}(\mathrm{~b})$, and the Hall conductivity $\sigma_{x y}$ (c) of a monolayer graphene calculated in the self-consistent Born approximation. The broadening of the Landau level $n$ becomes $\hbar \omega_{B} \sqrt{2 W\left(1+\delta_{n 0}\right)}$ and the peak value of $\sigma_{x x}$ becomes $\left(|n|+\delta_{n 0}\right)\left(e^{2} / \pi^{2} \hbar\right)$. The conductivity at $\varepsilon=0$ remains $e^{2} / \pi^{2} \hbar$ independent of the magnetic field. The Hall conductivity in the gap between adjacent Landau levels is quantized into $4(j+1 / 2)\left(e^{2} / h\right)$ with integer $j$.

self-consistent Born approximation. It is difficult to discuss this conductivity in the vicinity of zero energy, because a self-consistent determination of the screening and the density of states is necessary and because effects of higher-order scattering are likely to be important. The behavior near $\varepsilon \approx 0$ is expected to remain as an interesting and challenging subject.

Effects of level broadening due to disorder can be 
incorporated into the singular magnetic susceptibility discussed in the previous section. In fact, in a self-consistent Born approximation the delta-function changes into a smooth function with a sharp peak at $\varepsilon=0$ with a long tail decaying in proportion to $|\varepsilon|^{-1}$ with increasing $|\varepsilon|$ [37]. In a bilayer graphene disorder effects can simply be included by replacing $|\varepsilon|$ with $\sqrt{\varepsilon^{2}+\delta^{2}}$ with a phenomenological parameter $\delta$ because the susceptibility exhibits only a weaker logarithmic singularity as mentioned above and the density of states is independent of energy near $\varepsilon=0$.

\section{Screening Effect and Impurity Scattering}

The experiments further show that the conductivity does not exhibit a saturation with the increase of $n_{\mathrm{s}}$ and increases almost in proportion to $n_{\mathrm{s}}[11,12]$. This indicates that the effective strength of dominant scatterers decreases with $n_{\mathrm{s}}$ in actual graphenes, i.e., $W \propto$ $n_{\mathrm{s}}^{-1}$. A possible candidate is charged impurities giving rise to a long-range potential, for which the screening effect is known to play a vital role. It turns out that the screening property in graphenes exhibits behavior significantly different from that in conventional twodimensional systems [16].

The static dielectric function is written as

$$
\varepsilon(\mathbf{q})=1+\frac{2 \pi e^{2}}{\kappa_{0} q} \Pi(q)
$$

where the polarization function is given by

$$
\Pi(q)=-\frac{g_{\mathrm{v}} g_{\mathrm{s}}}{L^{2}} \sum_{s, s^{\prime}, \mathbf{k}}\left(f_{s \mathbf{k}}-f_{s^{\prime} \mathbf{k}+\mathbf{q}}\right) \frac{\left|\left(\mathbf{F}_{s \mathbf{k}}^{\dagger} \cdot \mathbf{F}_{s^{\prime} \mathbf{k}+\mathbf{q}}\right)\right|^{2}}{\varepsilon_{s \mathbf{k}}-\varepsilon_{s^{\prime} \mathbf{k}+\mathbf{q}}},
$$

where $f_{s \mathbf{k}}$ is the Fermi distribution function. Because the system is isotropic, the polarization function is determined by $q \equiv|\mathbf{q}|$, as can easily be proved by the above expression.

First, we define $\Pi^{0}(q)$ which is obtained from $\Pi(q)$ by replacing $f_{s \mathbf{k}}$ with $f_{s \mathbf{k}}^{0}$, where $f_{s \mathbf{k}}^{0}=1$ for $s=-1$ and 0 for $s=+1$. This $\Pi^{0}(q)$ describes effects of polarization of electrons in the valence band due to virtual interband transitions into the conduction band. It is calculated as $\Pi^{0}(q)=g_{\mathrm{v}} g_{\mathrm{s}} q / 16 \gamma$ as has been obtained previously $[38,39]$.

Because this polarization function is proportional to $q$, it's effect can be incorporated as the renormalization of the effective dielectric constant $\kappa_{0}$ to $\kappa$, with

$$
\frac{\kappa}{\kappa_{0}}=1+g_{\mathrm{v}} g_{\mathrm{s}} \frac{\pi^{2}}{4} U_{0}
$$

where the dimensionless interaction parameter $U_{0}=$ $e^{2} / 2 \pi \kappa_{0} \gamma$. For the band parameter $\gamma=6.46 \mathrm{eV} \cdot \AA$, corresponding to $\gamma_{0}=3.03 \mathrm{eV}$, we have $U_{0}=0.3545 / \kappa_{0}$. For $\kappa_{0} \approx 2.5$, we have $U_{0} \approx 0.14$, giving $\kappa / \kappa_{0} \approx 2.4$ or $\kappa \approx 6$. Correspondingly, the renormalized interaction parameter defined by $U=e^{2} / 2 \pi \kappa \gamma$ becomes $U \approx 0.06$. Effects of the interband polarization can be fully included by using these $\kappa$ and $U$ instead of $\kappa_{0}$ and $U_{0}$.

In the following we shall confine ourselves to the case that the Fermi level lies in the conduction band because of the symmetry between the conduction and valence bands. Define

$$
\tilde{f}_{s \mathbf{k}}=f_{s \mathbf{k}}-f_{s \mathbf{k}}^{0}
$$

Then, we have

$$
\Pi(q)=-2 \frac{g_{\mathrm{v}} g_{\mathrm{s}}}{L^{2}} \sum_{s, s^{\prime}, \mathbf{k}} \tilde{f}_{s \mathbf{k}} \frac{\left|\left(\mathbf{F}_{s, \mathbf{k}}^{\dagger} \cdot \mathbf{F}_{s^{\prime}, \mathbf{k}+\mathbf{q}}\right)\right|^{2}}{\varepsilon_{s \mathbf{k}}-\varepsilon_{s^{\prime} \mathbf{k}+\mathbf{q}}} .
$$

Performing the summation over $s^{\prime}= \pm 1$, we have

$\Pi(q)=-2 \frac{g_{\mathbf{v}} g_{\mathbf{s}}}{L^{2}} \sum_{\mathbf{k}} f^{+}\left(\varepsilon_{\mathbf{k}}\right) \frac{\gamma|\mathbf{k}|+\gamma|\mathbf{k}+\mathbf{q}| \cos \left(\theta_{\mathbf{k}}-\theta_{\mathbf{k + q}}\right)}{\gamma^{2}|\mathbf{k}|^{2}-\gamma^{2}|\mathbf{k}+\mathbf{q}|^{2}}$,

with

$$
f^{+}\left(\varepsilon_{\mathbf{k}}\right) \equiv \sum_{s= \pm 1} s \tilde{f}_{s \mathbf{k}}=f\left(\varepsilon_{\mathbf{k}}\right)+f\left(\varepsilon_{\mathbf{k}}+2 \zeta\right) .
$$

The integration over the direction of $\mathbf{q}$ can be performed easily and we have

$$
\Pi(q)=\frac{g_{\mathrm{v}} g_{\mathrm{s}}}{2 \pi \gamma}\left[\int_{0}^{\infty} f^{+}\left(\varepsilon_{\mathbf{k}}\right) d k-\int_{0}^{q / 2} f^{+}\left(\varepsilon_{\mathbf{k}}\right) \sqrt{1-\left(\frac{2 k}{q}\right)^{2}} d k\right] .
$$

At the absolute zero of temperature, we have $f^{+}(\varepsilon)=1$ for $\varepsilon<\varepsilon_{\mathrm{F}}$ and 0 for $\varepsilon>\varepsilon_{\mathrm{F}}$. For $q<2 k_{\mathrm{F}}$, therefore, we have

$$
\Pi(q)=\frac{g_{\mathrm{v}} g_{\mathrm{s}} k_{\mathrm{F}}}{2 \pi \gamma}\left(1-\frac{\pi}{4} \frac{q}{2 k_{\mathrm{F}}}\right),
$$

and for $q>2 k_{\mathrm{F}}$, on the other hand, we have

$$
\Pi(q)=\frac{g_{\mathrm{v}} g_{\mathrm{s}} k_{\mathrm{F}}}{2 \pi \gamma}\left[1-\frac{1}{2} \sqrt{1-\left(\frac{2 k_{\mathrm{F}}}{q}\right)^{2}}-\frac{1}{2} \frac{q}{2 k_{\mathrm{F}}} \sin ^{-1} \frac{2 k_{\mathrm{F}}}{q}\right],
$$

with the Fermi wavenumber $k_{\mathrm{F}}=\varepsilon_{\mathrm{F}} / \gamma$. This behavior is quite different from that in conventional twodimensional metals where the polarization function is constant for $q<2 k_{\mathrm{F}}$ and decreases for $q>2 k_{\mathrm{F}}$ with a divergent first derivative at $q=2 k_{\mathrm{F}}[9]$. 


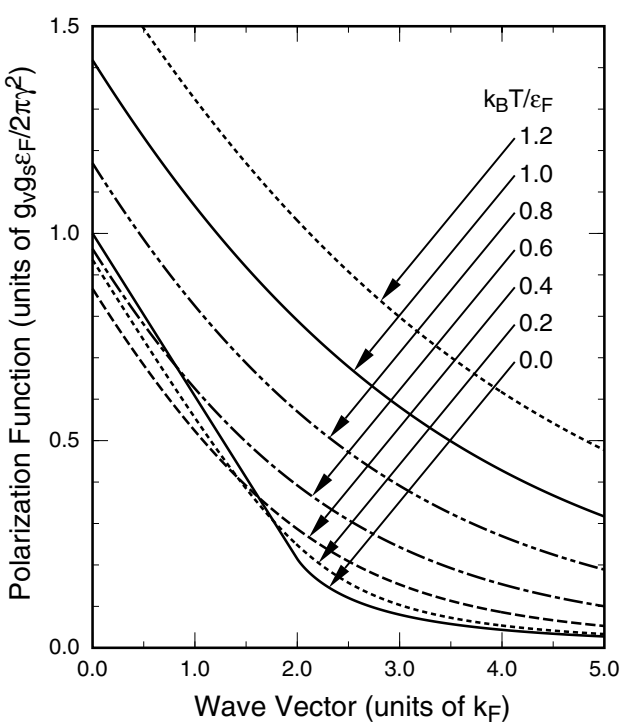

Fig. 7. The polarization function as a function of the wave vector at different temperatures. It does not exhibit an apparent singularity at $q / k_{\mathrm{F}}=2$ even at zero temperature.

The reason lies in the vanishing Coulomb matrix element for the backward scattering $\mathbf{k} \rightarrow-\mathbf{k}$. The polarization function is dominated by virtual transitions between states with similar energy because of small energy denominator. Such energy conserving processes are present only for $q<2 k_{\mathrm{F}}$ and disappear suddenly at $q=2 k_{\mathrm{F}}$. This singular behavior is known to be the origin of the large Kohn anomaly in two-dimensional systems [9]. In graphene, the contributions of energy conserving processes become weaker with the increase of $q$ even for $q<2 k_{\mathrm{F}}$ because of the decrease of the matrix element for large angle scattering, leading to the decrease of $\Pi(q)$ for $q<2 k_{\mathrm{F}}$. At $q=2 k_{\mathrm{F}}$, the matrix element of energy conserving processes vanishes identically due to the absence of backscattering and therefore no strong singular behavior can appear at $q=2 k_{\mathrm{F}}$.

In the long-wavelength limit, the dielectric function is written as

$$
\varepsilon(q)=1+\frac{q_{\mathrm{s}}}{q},
$$

where $q_{\mathrm{s}}$ is called the screening constant given by

$$
q_{\mathrm{s}}=\frac{2 \pi e^{2}}{\kappa} \Pi(0) .
$$

It is easily shown that

$$
\Pi(0)=\int_{-\infty}^{\infty}\left(-\frac{\partial f(\varepsilon)}{\partial \varepsilon}\right) D(\varepsilon) d \varepsilon,
$$

which is known as the compressibility sum-rule.

The transport relaxation time for energy $\varepsilon(\varepsilon>0)$ is given by

$$
\frac{\hbar}{\tau(\varepsilon)}=2 \pi n_{\mathrm{i}} D(\varepsilon) \int_{0}^{\pi} \frac{d \theta}{\pi} \frac{1}{2}\left(1-\cos ^{2} \theta\right)\left(\frac{2 \pi e^{2}}{\kappa q \varepsilon(q)}\right)^{2} .
$$

where $n_{\mathrm{i}}$ is the concentration of impurities and $q=$ $2(\varepsilon / \gamma) \sin (\theta / 2)$ in the integrand. This equation shows that the probability of backward scattering $\theta=\pi$ vanishes identically, which corresponds to the absence of backscattering in metallic carbon nanotubes $[4,26]$.

The relaxation time can be rewritten as

$$
\tau(\varepsilon)=\frac{n_{\mathrm{s}}}{\pi g_{\mathrm{v}} g_{\mathrm{s}} n_{\mathrm{i}}} \frac{\hbar}{|\varepsilon|} H(\varepsilon),
$$

with the dimensionless quantity $H(\varepsilon)$ defined by

$$
H(\varepsilon)^{-1}=\int_{0}^{\pi} \frac{d \theta}{\pi} \frac{1}{2}\left(1-\cos ^{2} \theta\right)\left(\frac{1}{2 \pi U} \frac{\gamma q}{\varepsilon_{\mathrm{F}}}+g_{\mathrm{v}} g_{\mathrm{s}} \tilde{\Pi}(q)\right)^{-2},
$$

where $\tilde{\Pi}(q)$ is the dimensionless quantity defined by

$$
\Pi(q) \equiv \frac{g_{\mathrm{v}} g_{\mathrm{s}} \varepsilon_{\mathrm{F}}}{2 \pi \gamma^{2}} \tilde{\Pi}(q) .
$$

At zero temperature, $H_{0} \equiv H\left(\varepsilon_{\mathrm{F}}\right)$ is independent of the electron concentration and determined by $U$ alone. The mobility becomes

$$
\mu_{0}=\frac{e}{4 \pi^{2} \hbar n_{\mathrm{i}}} H_{0},
$$

which is independent of $n_{\mathrm{s}}$. The conductivity becomes

$$
\sigma_{0}=\frac{e^{2}}{4 \pi^{2} \hbar} \frac{n_{\mathrm{s}}}{n_{\mathrm{i}}} H_{0}
$$

which is proportional to $n_{\mathrm{s}}$. For $U \approx 0.06$, we have $H_{0} \approx$ 120 .

The observed value at high $n_{\mathrm{s}}$ close to $\mu \sim 10^{4}$ $\mathrm{cm}^{2} / \mathrm{Vs}$ [12] corresponds to the impurity concentration of $n_{\mathrm{i}} \sim 4 \times 10^{11} \mathrm{~cm}^{-2}$. Such amount of impurities are highly likely to be present on a substrate surface. It is interesting that this is comparable to the concentration of charged impurities present in the vicinity of the $\mathrm{Si}$ and $\mathrm{SiO}_{2}$ interface in typical MOSFET's [9]. Effects of charged-impurity scattering were also discussed within a less accurate Thomas-Fermi approximation where $\Pi(q)$ is replaced by $\Pi(0)$ [40]. It was suggested also that the linear screening may not be valid near $\varepsilon \approx 0$ [41]. 


\section{Symmetry Crossover}

The Weyl equation (2) is invariant under a special time-reversal operation $S$,

$$
\mathbf{F}^{S}=K \mathbf{F}^{*},
$$

where $\mathbf{F}^{*}$ represents the complex conjugate of the wave function $\mathbf{F}$ and $K$ is an anti-unitary matrix $K=-i \sigma_{y}$ satisfying $K^{2}=-1$. The corresponding operation for an operator $P$ is given by $P^{S}=K^{t} P K^{-1}$, where ${ }^{t} P$ stands for the transpose of $P$. This corresponds to the time reversal in systems with spin-orbit interaction and leads to

$$
\mathbf{F}^{S^{2}} \equiv\left(\mathbf{F}^{S}\right)^{S}=-\mathbf{F} .
$$

The system belongs to the symplectic universality class when only $S$ constitutes a relevant symmetry [42].

This symmetry prevails even in the presence of impurities unless their potential range is smaller than the lattice constant $a$. In fact, for such scatterers, the effective potential is the same for the A and B sites and does not cause any mixing between the $\mathrm{K}$ and $\mathrm{K}$ ' points. In this case a quantum correction or a weaklocalization correction to the Boltzmann conductivity becomes positive and diverges logarithmically [7]. This so-called anti-localization behavior is the same as that appears in systems with strong spin-orbit interaction.

The operation $S$ is not the real time-reversal in graphene. Actually, the Bloch functions at the $\mathrm{K}$ and $\mathrm{K}$ ' points are mutually complex conjugate and therefore, the $\mathrm{K}$ point is converted into the $\mathrm{K}$ ' point and the $K^{\prime}$ point into the $\mathrm{K}$ point under the real time reversal. In the present $\mathbf{k} \cdot \mathbf{p}$ scheme, this operation $T$ is expressed as

$$
\mathbf{F}_{K}^{T}=\mathrm{e}^{-i \psi} \sigma_{z} \mathbf{F}_{K^{\prime}}^{*}, \quad \mathbf{F}_{K^{\prime}}^{T}=\mathrm{e}^{-i \psi} \sigma_{z} \mathbf{F}_{K}^{*},
$$

where $\psi$ is an arbitrary phase factor and $\mathbf{F}_{K}$ and $\mathbf{F}_{K^{\prime}}$ are the wave functions at the $\mathrm{K}$ and $\mathrm{K}^{\prime}$ points, respectively. This immediately gives

$$
\mathbf{F}_{K}^{T^{2}} \equiv\left(\mathbf{F}_{K}^{T}\right)^{T}=\mathbf{F}_{K}, \quad \mathbf{F}_{K^{\prime}}^{T^{2}} \equiv\left(\mathbf{F}_{K^{\prime}}^{T}\right)^{T}=\mathbf{F}_{K^{\prime}},
$$

characteristic of the conventional orthogonal symmetry. When we can neglect mixing between the $\mathrm{K}$ and $\mathrm{K}^{\prime}$ points and confine ourselves to states in each valley, however, the $T$ symmetry is irrelevant and the special $S$ symmetry becomes relevant. In the presence of short-range scatterers causing mixing between $\mathrm{K}$ and K' points, the $S$ symmetry is violated, but the $T$ sym-

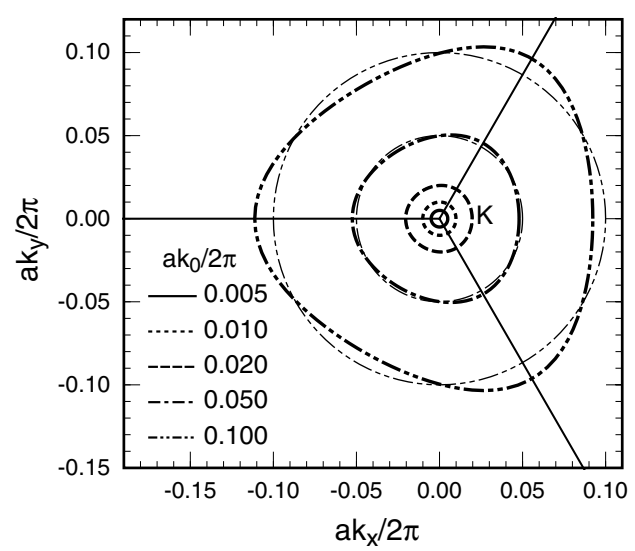

Fig. 8. Some examples of equi-energy lines in the vicinity of the $\mathrm{K}$ point in monolayer graphene.

metry prevails. As a result the system now belongs to the orthogonal class $[7,43]$.

The actual equi-energy line deviates from the circle and has trigonal warping when the energy becomes nonzero as shown in Fig. 8. This effect can be included by a higher order $\mathbf{k} \cdot \mathbf{p}$ term. For the $\mathrm{K}$ point it is given by

$$
\begin{aligned}
& H_{1}=\gamma\left(\begin{array}{cc}
0 & h_{1}\left(\hat{k}_{x}, \hat{k}_{y}\right) \\
h_{1}\left(\hat{k}_{x}, \hat{k}_{y}\right)^{\dagger} & 0
\end{array}\right), \\
& h_{1}\left(\hat{k}_{x}, \hat{k}_{y}\right)=\alpha \frac{a}{4 \sqrt{3}}\left(\hat{k}_{x}+i \hat{k}_{y}\right)^{2}
\end{aligned}
$$

where $\alpha$ is a constant of the order of unity. This higher order term gives rise to a trigonal warping of the dispersion. This expression with $\alpha=1$ has been derived from a nearest-neighbor tight-binding model [44], but is much more general if we regard $\beta$ as an adjustable parameter. In the presence of $H_{1}$, the special time reversal symmetry is destroyed because $H_{1}^{S}=-H_{1}$ [45]. As a result the system now belongs to the unitary class. For the K' point, the effective Hamiltonian is obtained by the replacement of $h_{1}$ by $-h_{1}^{\dagger}$.

It should be noted also that the presence of lattice strain gives rise to an effective vector potential and therefore destroys the $S$ symmetry [46-49]. In fact, the effective Hamiltonian in the presence of longwavelength lattice displacement $\mathbf{u}(\mathbf{r})=\left(u_{x}, u_{y}\right)$ is given by

$$
H_{2}=g_{1}\left(u_{x x}+u_{y y}\right)+g_{2}\left[\left(u_{x x}-u_{y y}\right) \sigma_{x}-2 u_{x y} \sigma_{y}\right],
$$

for the $\mathrm{K}$ point, where the strain tensor is given by 


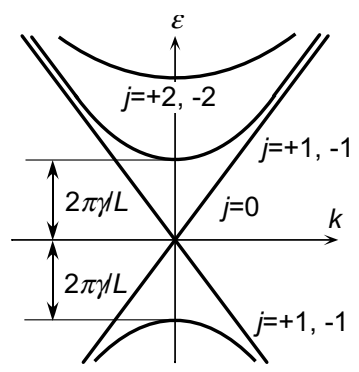

Fig. 9. Energy bands of a metallic carbon nanotube with circumference $L$ obtained in a $\mathbf{k} \cdot \mathbf{p}$ scheme.

$$
u_{x x}=\frac{\partial u_{x}}{\partial x}, u_{y y}=\frac{\partial u_{y}}{\partial y}, u_{x y}=\frac{1}{2}\left(\frac{\partial u_{x}}{\partial y}+\frac{\partial u_{y}}{\partial x}\right) \text {. }
$$

The interaction strength $g_{1}$ is called the deformation potential ( $\sim 20 \mathrm{eV}$ in bulk graphite) and $g_{2} \approx \beta \gamma_{0} / 4$ appears due to change in the bond length, where the dimensionless parameter $\beta$ is given by

$$
\beta=-\frac{d \ln \gamma_{0}}{d \ln b}
$$

where $b$ is the bond length of nearest-neighbor carbon atoms given by $b=a / \sqrt{3}$ [49]. The Hamiltonian for the $\mathrm{K}$ ' point is obtained by replacing $g_{2}$ by $-g_{2}$ and $\boldsymbol{\sigma}$ by $\boldsymbol{\sigma}^{*}$. A nonzero curvature also gives rise to an effective vector potential and destroys the $S$ symmetry $[46,50]$.

The time reversal symmetry is known to manifest itself as a quantum correction to the conductivity. If the system has the $S$ symmetry, quantum correction $\Delta \sigma$ given by so-called maximally crossed diagrams exhibits an anti-localization behavior, i.e., the conductivity increases logarithmically with the decrease of the temperature. If the system has only $T$ symmetry, $\Delta \sigma$ exhibits a weak-localization behavior and the conductivity decreases logarithmically with the temperature decrease. The crossover between the weak- and antilocalization behavior caused by short-range scatterers was demonstrated [7]. Suppression of weak localization observed in graphene [23] was analyzed by the crossover among symplectic, orthogonal, and unitary due to various perturbations mentioned above [20].

The symmetry plays important roles in carbon nanotubes [4]. Figure 9 shows the energy bands in a metallic nanotube with circumference $L$, where the wave vector along the circumference is quantized into $(2 \pi / L) j$ with integer $j$. In the presence of the $S$ symmetry, the reflection coefficients satisfy the symmetry relation $r_{\bar{\alpha} \beta}=-r_{\bar{\beta} \alpha}$, where $\alpha$ and $\beta$ denote in-coming channels and $\bar{\alpha}$ and $\bar{\beta}$ their time reversal or out-going channels.
Metallic nanotubes satisfy this symmetry and possess an odd number of current carrying channels for each of the $\mathrm{K}$ and $\mathrm{K}$ ' points. When only a single conducting channel is present, we have $r_{\bar{\alpha} \alpha}=0$ and the conductance is given by an ideal value $2 e^{2} / \pi \hbar[26,27]$.

The determinant of an antisymmetric matrix with odd dimension is known to vanish identically. By definition, $r_{\bar{\beta} \alpha}$ represents the amplitude of a reflective wave $\bar{\beta}$ with wave function $\psi_{\bar{\beta}}(\mathbf{r})$ corresponding to an in-coming wave $\alpha$ with wave function $\psi_{\alpha}(\mathbf{r})$. The vanishing determinant of $r$ shows that there exists at least one nontrivial solution for the equation

$$
\sum_{\alpha=1}^{n_{\mathrm{c}}} r_{\bar{\beta} \alpha} a_{\alpha}=0
$$

Then, there is no reflected wave for the incident wave $\sum_{\alpha} a_{\alpha} \psi_{\alpha}(\mathbf{r})$. Therefore, this becomes a perfectly conducting channel which transmits through the system without being scattered back [51].

An interesting feature of nanotubes is the AharonovBohm (AB) effect on the band structure [25,52]. Recently, the splitting of optical absorption and emission peaks due to flux was observed [53-55]. In the presence of a magnetic flux the time-reversal symmetry is absent and therefore the perfectly conducting channel is destroyed. Explicit numerical calculations [56] showed that the perfect channel is quite fragile and disappears for flux less than $1 \%$ of the flux quantum $\phi_{0}=\mathrm{ch} / \mathrm{e}$, while the absence of backscattering in linear bands is quite robust against such perturbations.

When the flux is a half of the flux quantum, the time-reversal symmetry is recovered but the channel number is even. In this case, the determinant of an antisymmetric matrix does not vanish and the perfect channel is destroyed. Therefore, the flux dependence in nanotubes is quite different from a $\phi_{0} / 2$ oscillation in metallic systems on a cylinder surface, predicted theoretically [57] and observed experimentally [58]. This AAS oscillation arises due to the symmetry change caused by the flux with period $\phi_{0} / 2$.

The symmetry crossover due to magnetic field [56], short-range scatterers [43], and trigonal warping [45] manifests itself in the flux dependence of the localization effect in metallic carbon nanotubes [59]. Figure 10 compares the inverse localization length as a function of the flux, (a) when the system has the $S$ symmetry, (b) when the $S$ symmetry is destroyed by a strong trigonal warping, and (c) when the system has only the $T$ 

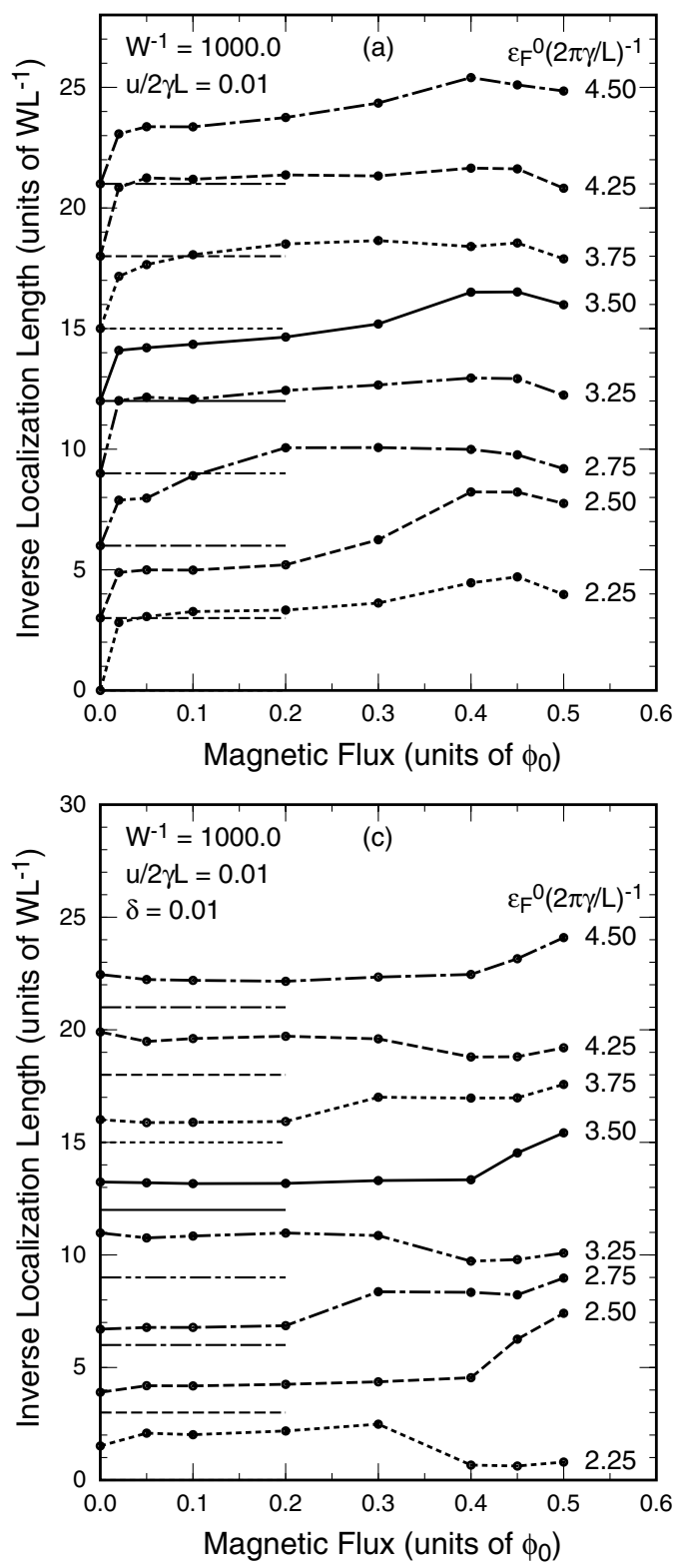

symmetry due to short-range scatterers. Quantum correction to the conductivity was also studied in metallic carbon nanotubes [60], but turned out to be not so successful in describing the features demonstrated in the numerical results. This absence of backscattering and the presence of a perfect channel lead also to intriguing behavior in the dynamical conductivity [61,62]. Further, strong short-range scatterers like lattice vacancies [63-66] and topological deffects like five- and seven-

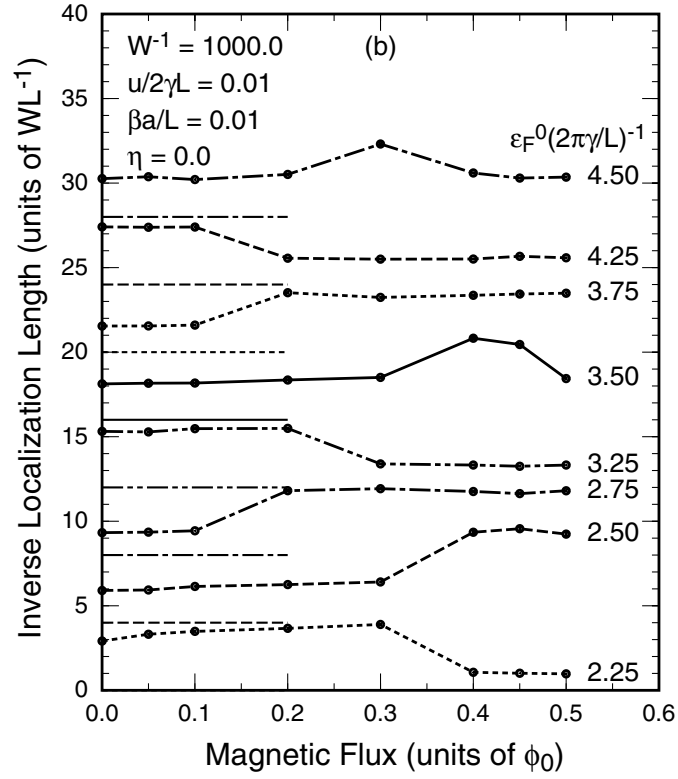

Fig. 10. Calculated inverse localization length as a function of flux $\phi$ in a metallic carbon nanotube. (a) Scatterers with a potential range larger than the lattice constant. (b) Effects of trigonal warping. (c) Scatterers with short-range potential. In (a) the inverse localization length vanishes identically for $\phi=0$ due to the presence of a perfectly conducting channel. The localization effect appears for nonzero $\phi$ due to the symmetry crossover to the unitary, but takes a local minimum at the half flux quantum where the symplectic symmetry is recovered but states are localized because the channel number is even. In (b) the system has the unitary symmetry independent of the flux and the localization effect is essentially independent of the flux. In (c) the localization effect becomes weaker for nonzero flux except at the half flux quantum.

membered rings $[67,68]$ and a Stone-Wales defect [69] also cause a singular scattering behavior.

\section{Anomaly in Optical Phonon}

Long-wavelength phonons are known to be measured directly by the Raman scattering [70,71]. Usu- 
ally, they are described perfectly well in a continuum model. A a correct description of long-wavelength acoustic phonons was constructed and applied to carbon nanotubes [49] and a similar continuum model was developed for optical phonons and the Hamiltonian for electron-phonon interactions was derived also [72]. With the use of such continuum model effects of electron-phonon interaction on optical phonons was recently studied theoretically [73,74].

Optical phonons are represented by the relative displacement of two sub-lattice atoms A and B,

$$
\mathbf{u}(\mathbf{r})=\sum_{\mathbf{q}, \mu} \sqrt{\frac{\hbar}{2 N M \omega_{0}}}\left(b_{\mathbf{q} \mu}+b_{-\mathbf{q} \mu}^{\dagger}\right) \mathbf{e}_{\mu}(\mathbf{q}) \mathrm{e}^{i \mathbf{q} \cdot \mathbf{r}},
$$

where $N$ is the number of unit cells, $M$ is the mass of a carbon atom, $\omega_{0}$ is the phonon frequency at the $\Gamma$ point, $\mathbf{q}=\left(q_{x}, q_{y}\right)$ is the wave vector, $\mu$ denotes the modes ( $\mathrm{t}$ for transverse and $\mathrm{l}$ for longitudinal), and $b_{\mathbf{q} \mu}^{\dagger}$ and $b_{\mathbf{q} \mu}$ are the creation and destruction operators, respectively. Define $q_{x}=q \cos \varphi_{\mathbf{q}}$ and $q_{y}=q \sin \varphi_{\mathbf{q}}$ with $q=|\mathbf{q}|$. Then, we have

$$
\begin{aligned}
& \mathbf{e}_{\mathrm{l}}(\mathbf{q})=i\left(\cos \varphi_{\mathbf{q}}, \sin \varphi_{\mathbf{q}}\right), \\
& \mathbf{e}_{\mathrm{t}}(\mathbf{q})=i\left(-\sin \varphi_{\mathbf{q}}, \cos \varphi_{\mathbf{q}}\right) .
\end{aligned}
$$

The corresponding phonon Hamiltonian is written as

$$
H_{\mathrm{ph}}=\sum_{\mathbf{q}, \mu} \hbar \omega_{0}\left(b_{\mathbf{q} \mu}^{\dagger} b_{\mathbf{q} \mu}+\frac{1}{2}\right) .
$$

The interaction between optical phonons and an electron at the $\mathrm{K}$ point is given by [72]

$$
H_{\mathrm{int}}^{\mathrm{K}}=-\sqrt{2} \frac{\beta \gamma}{b^{2}} \boldsymbol{\sigma} \times \mathbf{u}(\mathbf{r}),
$$

where the vector product for vectors $\mathbf{a}=\left(a_{x}, a_{y}\right)$ and $\mathbf{b}=\left(b_{x}, b_{y}\right)$ in two dimension is defined by $\mathbf{a} \times \mathbf{b}=$ $a_{x} b_{y}-a_{y} b_{x}$. The Hamiltonian for the K' point is obtained by replacing $\boldsymbol{\sigma}$ by $\boldsymbol{\sigma}^{*}$. This means that the lattice distortion gives rise to a shift in the origin of the wave vector or an effective vector potential, i.e., $u_{x}$ in the $y$ direction and $u_{y}$ in the $x$ direction.

The phonon Green's function is written as

$$
D_{\mu}(\mathbf{q}, \omega)=\frac{2 \hbar \omega_{0}}{(\hbar \omega)^{2}-\left(\hbar \omega_{0}\right)^{2}-2 \hbar \omega_{0} \Pi_{\mu}(\mathbf{q}, \omega)} .
$$

In the following, we shall consider retarded Green's function obtained by an analytic continuation of a thermal Green's function from upper complex plane. The phonon frequency is determined by the pole of $D_{\mu}(\mathbf{q}, \omega)$ as

$$
\left(\frac{\omega}{\omega_{0}}\right)^{2}-1=\frac{2}{\hbar \omega_{0}} \operatorname{Re} \Pi_{\mu}(\mathbf{q}, \omega) .
$$



Fig. 11. The frequency shift (solid line) and broadening (dashed line) of optical phonons as a function of the Fermi energy. $\tau$ is a phenomenological relaxation time characterizing the level broadening effect due to disorder.

As will become clear in the following, the phonon selfenergy is small. In this case, the shift of the phonon frequency is given by

$$
\Delta \omega_{\mu}=\frac{1}{\hbar} \operatorname{Re} \Pi_{\mu}\left(\mathbf{q}, \omega_{0}\right),
$$

and the broadening is given by

$$
\Gamma_{\mu}=-\frac{1}{\hbar} \operatorname{Im} \Pi_{\mu}\left(\mathbf{q}, \omega_{0}\right) .
$$

Define the dimensionless coupling parameter

$$
\lambda=\frac{g_{\mathrm{v}} g_{\mathrm{s}}}{4} \frac{36 \sqrt{3}}{\pi} \frac{\hbar^{2}}{2 M a^{2}} \frac{1}{\hbar \omega_{0}}\left(\frac{\beta}{2}\right)^{2} .
$$

For $M=1.993 \times 10^{-23} \mathrm{~g}$ and $\hbar \omega_{0}=0.196 \mathrm{eV}$, we have

$$
\lambda \approx 3 \times 10^{-3} \frac{g_{\mathrm{v}} g_{\mathrm{s}}}{4}\left(\frac{\beta}{2}\right)^{2} .
$$

This parameter is slightly different from that defined in ref. [72]. Then, we have

$$
\begin{aligned}
\Pi_{\mu}(\mathbf{q}, \omega) & =-\lambda \int_{0}^{\infty} \varepsilon d \varepsilon[f(-\varepsilon)-f(\varepsilon)] \\
& \times\left(\frac{1}{\hbar \omega+2 \varepsilon+i 0}-\frac{1}{\hbar \omega-2 \varepsilon+i 0}\right),
\end{aligned}
$$

independent of the phonon mode.

We are calculating the self-energy of optical phonons starting with the known phonon modes in graphene. 


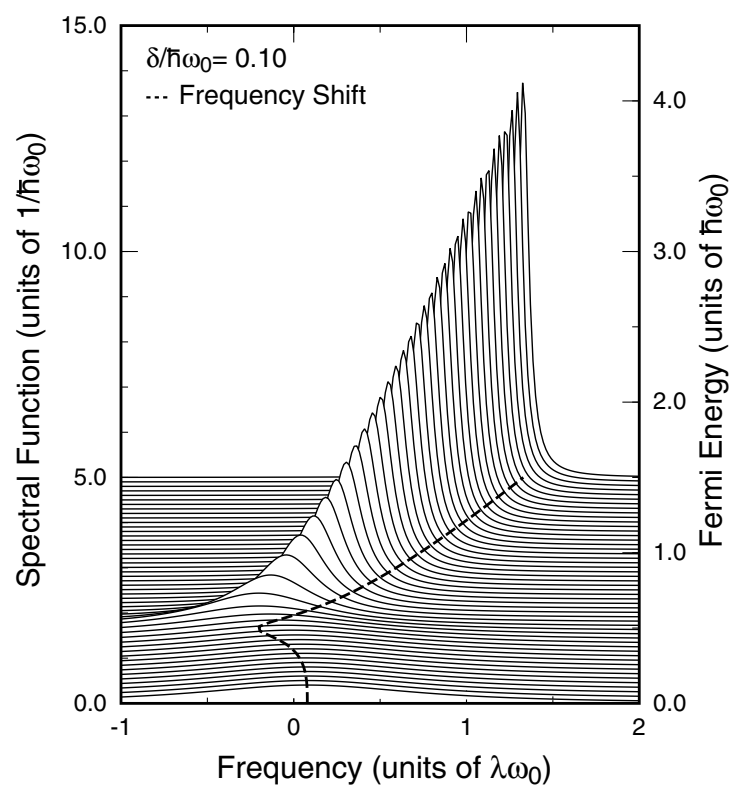

Fig. 12. Calculated spectral function of optical phonon for varying Fermi energy.

Therefore, the direct evaluation of the above selfenergy causes a problem of double counting [75]. In fact, if we apply the above formula to the case of vanishing Fermi energy, we get the frequency shift due to virtual excitations of all electrons in the $\pi$ bands. However, this contribution is already included in the definition of the frequency $\omega_{0}$. In order to avoid such a problem, we have to subtract the contribution in the undoped graphene for $\omega=0$ corresponding to the adiabatic approximation.

This contribution can be obtained from the above by putting $\omega=0$ and $f(\varepsilon)=f_{0}(\varepsilon)$, with $f_{0}=0$ for $\varepsilon>0$ and 1 for $\varepsilon<0$ as

$$
\Pi_{\mu}^{2 \mathrm{D}}(\mathbf{q}, \omega)=-\lambda \int_{0}^{\varepsilon_{\mathrm{c}}} \varepsilon d \varepsilon\left(\frac{1}{2 \varepsilon+i 0}+\frac{1}{2 \varepsilon-i 0}\right)=-\lambda \varepsilon_{\mathrm{c}}
$$

where we have introduced the cutoff energy $\varepsilon_{\mathrm{c}}$ of the order of the half of the $\pi$-band width. Thus, the redefined self-energy becomes

$$
\begin{aligned}
\Pi_{\mu}(\mathbf{q}, \omega) & =\lambda \int_{0}^{\infty} d \varepsilon[1-f(-\varepsilon)+f(\varepsilon)] \\
& +\frac{1}{2} \lambda(\hbar \omega+i \delta) \int_{0}^{\infty} d \varepsilon[f(-\varepsilon)-f(\varepsilon)] \\
& \times\left(\frac{1}{\hbar \omega+2 \varepsilon+i \delta}+\frac{1}{\hbar \omega-2 \varepsilon+i \delta}\right)
\end{aligned}
$$

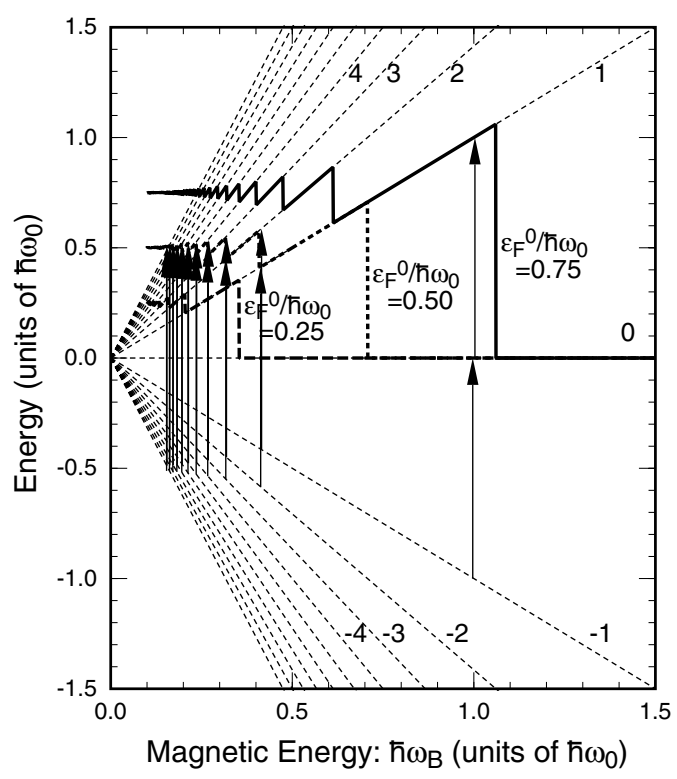

Fig. 13. The Landau levels (thin dotted lines) as a function of effective magnetic energy $\hbar \omega_{B} \propto \sqrt{B}$ and the Fermi energy for a given electron concentration characterized by the Fermi energy $\varepsilon_{\mathrm{F}}^{0}$ in the absence of a magnetic field. The vertical arrows show transitions at a resonance.

where a phenomenological imaginary part $\delta=\hbar / \tau$ has been introduced as a parameter characterizing scattering of electrons due to disorder.

We have the relation $f_{-\zeta}(\varepsilon)=1-f_{\zeta}(-\varepsilon)$, which leads to the conclusion that the self-energy is symmetric between $\zeta>0$ and $\zeta<0$, i.e., the electron-hole symmetry. We shall confine ourselves to the case $\zeta>0$.

At zero temperature, in particular, we have

$\Pi_{\mu}(\mathbf{q}, \omega)=\lambda \varepsilon_{\mathrm{F}}-\frac{1}{4} \lambda(\hbar \omega+i \delta)\left(\ln \frac{\hbar \omega+2 \varepsilon_{\mathrm{F}}+i \delta}{\hbar \omega-2 \varepsilon_{\mathrm{F}}+i \delta}+\pi i\right)$,

where $\varepsilon_{\mathrm{F}}$ is the Fermi energy. In the clean limit $\delta \rightarrow 0$, we have

$\Pi_{\mu}(\mathbf{q}, \omega)=\lambda \varepsilon_{\mathrm{F}}-\frac{1}{4} \lambda \hbar \omega\left[\ln \left|\frac{\hbar \omega+2 \varepsilon_{\mathrm{F}}}{\hbar \omega-2 \varepsilon_{\mathrm{F}}}\right|+\pi i \theta\left(\hbar \omega-2 \varepsilon_{\mathrm{F}}\right)\right]$.

The frequency shift, i.e., the real part, diverges logarithmically to $-\infty$ at $\varepsilon_{\mathrm{F}}=\hbar \omega_{0} / 2$. Apart from this logarithmic singularity, the phonon frequency increases roughly in proportion to $\varepsilon_{\mathrm{F}}$ for $\varepsilon_{\mathrm{F}}>\hbar \omega_{0}$. The broadening is nonzero only for $\varepsilon_{\mathrm{F}}<\hbar \omega_{0} / 2$.

Figure 11 shows the frequency shift and broadening for various values of $1 / \omega_{0} \tau$. For nonzero $\delta$ or $1 / \omega_{0} \tau$, the logarithmic singularity of the frequency 


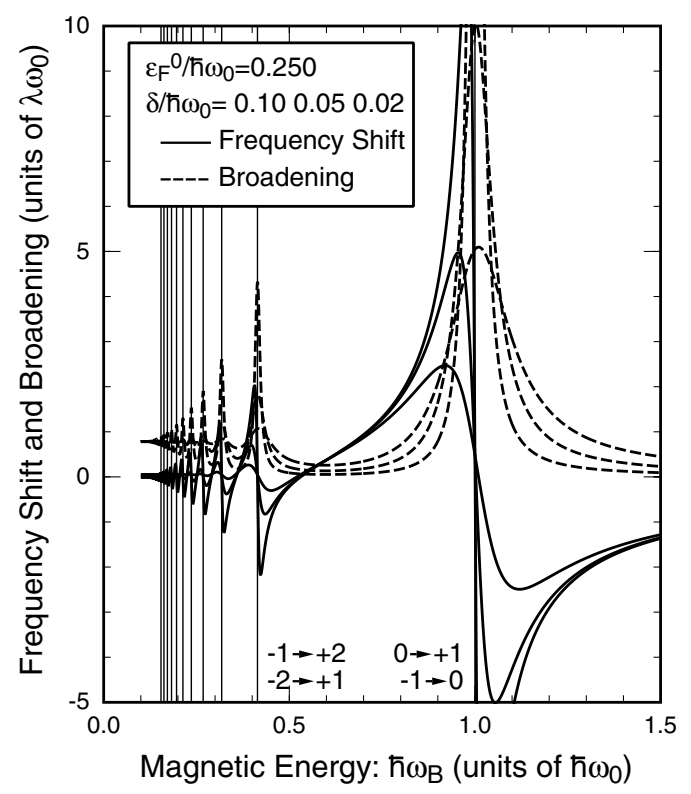

Fig. 14. The frequency shift (solid line) and broadening (dashed line) of optical phonons as a function of effective magnetic energy $\hbar \omega_{B}$ at low electron concentrations corresponding to inter-band resonances. Thin vertical lines show resonance magnetic fields. $\varepsilon_{\mathrm{F}}^{0} / \hbar \omega_{0}=1 / 4$. The results for $\delta / \hbar \omega_{0}=0.1,0.05$, and 0.02 are shown.

shift and the sharp drop in the broadening disappear, but the corresponding features remain for $1 / \omega_{0} \tau \ll 1$. Figure 12 shows the corresponding spectral function $(-1 / \pi) \operatorname{Im} D\left(q, \omega_{0}\right)$. Similar results were reported independently [76] and experiments giving qualitatively similar results were reported recently $[77,78]$.

The calculation can easily be extended to the case in the presence of a magnetic field, where discrete Landau levels are formed and oscillations due to resonant interactions appear in the frequency shift and the broadening [74]. The self-energy is calculated as

$$
\begin{gathered}
\Pi(\mathbf{q}, \omega)=-\frac{1}{4} \lambda\left(\hbar \omega_{B}\right)^{2} \sum_{s, s^{\prime}= \pm 1} \sum_{n=0}^{\infty}\left(\left[f\left(s \varepsilon_{n}\right)-f\left(s^{\prime} \varepsilon_{n+1}\right)\right]\right. \\
\left.\times \frac{2\left(s \varepsilon_{n}-s^{\prime} \varepsilon_{n+1}\right)}{(\hbar \omega+i \delta)^{2}-\left(s \varepsilon_{n}-s^{\prime} \varepsilon_{n+1}\right)^{2}}-\frac{1-s s^{\prime}}{\varepsilon_{n+1}+\varepsilon_{n}}\right) .
\end{gathered}
$$

This shows that a resonance behavior appears when $\hbar \omega_{B}^{n \pm}=\hbar \omega_{0}$ with

$$
\hbar \omega_{B}^{n \pm}=\hbar \omega_{B}(\sqrt{n+1} \pm \sqrt{n}) .
$$

The contribution of the resonance is given by

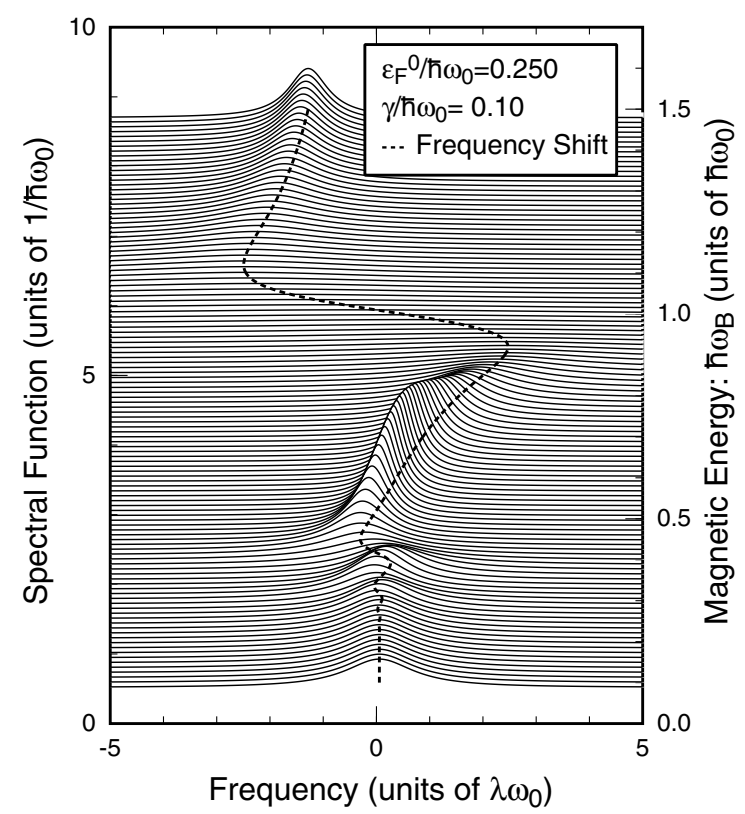

Fig. 15. The phonon spectral function $(-1 / \pi) \operatorname{Im} D(\mathbf{q}, \omega)$ for $\varepsilon_{\mathrm{F}}^{0} / \hbar \omega_{0}=1 / 4 . \delta / \hbar \omega_{0}=0.1$. The dotted line shows the frequency shift, i.e., the peak position, as a function of $\hbar \omega_{B}$.

$$
\frac{2 \hbar \omega_{B}^{n \pm}}{\left(\hbar \omega_{0}+i \delta\right)^{2}-\left(\hbar \omega_{B}^{n \pm}\right)^{2}} \approx \frac{1}{\hbar \omega_{0}-\hbar \omega_{B}^{n \pm}+i \delta}
$$

apart from a factor determined by the filling of the relevant Landau levels. The imaginary part has a Lorentzian peak and the real part exhibits a singularity $\sim\left(\omega_{0}-\omega_{B}^{n \pm}\right)^{-1}$.

Such resonances can be classified into inter-band processes $\omega_{B}^{n+} \approx \omega_{0}$ and intra-band processes $\omega_{B}^{n-} \approx \omega_{0}$. The latter appears only in high magnetic fields at sufficiently high electron concentrations and the former appears in weak magnetic fields at low electron concentrations. Figure 13 shows some Landau levels $\pm \hbar \omega_{B} \sqrt{n}$ as a function of the effective magnetic energy $\hbar \omega_{B}$, together with the Fermi energy for a given electron concentration specified by zero-field Fermi energy $\varepsilon_{F}^{0}$. The resonance fields are specified by arrows connecting initial and final states.

Figure 14 shows calculated frequency shift and broadening when $\varepsilon_{\mathrm{F}} / \hbar \omega_{0}=1 / 4$. All resonant transitions from $-n$ to $n+1$ and from $-n-1$ to $n$ appear at $\omega_{B}^{n+}=\hbar \omega_{0}$. Figure 15 shows the phonon spectral function $(-1 / \pi) \operatorname{Im} D\left(q, \omega_{0}\right)$. At resonances, the phonon spectrum exhibits characteristic behavior and the phonon practically disappears due to large broad- 
ening at a magnetic field corresponding to the exact resonance. Experimental observation of this magnetic oscillation of optical phonons is highly expected.

\section{Summary and Conclusion}

A brief review has been given on electronic and transport properties of graphene from a theoretical point of view. The topics have been the effective-mass description of electronic states, topological anomaly associated with Berry's phase, singular diamagnetic susceptibility, zero-mode anomalies and their removal due to level broadening effects, screening effect and charged impurity scattering, the symmetry crossover among symplectic, unitary, and orthogonal due to the presence of special time reversal symmetry, and anomaly and magnetic oscillation of long-wavelength optical phonons.

\section{Acknowledgments}

This work was supported in part by a 21 st Century COE Program at Tokyo Tech "Nanometer-Scale Quantum Physics" and by Grant-in-Aid for Scientific Research from the Ministry of Education, Culture, Sports, Science and Technology, Japan.

\section{References}

[1] J. W. McClure, Phys. Rev. 104, 666 (1956).

[2] J. C. Slonczewski and P. R. Weiss, Phys. Rev. 109, 272 (1958).

[3] T. Ando, Semicond. Sci. \& Technol. 15, R13 (2000); Solid State Commun. 12, 69 (2003); Physica E 20, 24 (2003); Physics E 22, 656 (2004).

[4] T. Ando, J. Phys. Soc. Jpn. 74, 777 (2005).

[5] N. H. Shon and T. Ando, J. Phys. Soc. Jpn. 67, 2421 (1998).

[6] Y. Zheng and T. Ando, Phys. Rev. B 65, 245420 (2002).

[7] H. Suzuura and T. Ando, Phys. Rev. Lett. 89, 266603 (2002).

[8] T. Ando, Y. Zheng, and H. Suzuura, J. Phys. Soc. Jpn. 71, 1318 (2002).
[9] T. Ando, A. B. Fowler, and F. Stern, Rev. Mod. Phys. 54, 437 (1982) and references cited therein.

[10] K. S. Novoselov, A. K. Geim, S. V. Morozov, D. Jiang, Y. Zhang, S. V. Dubonos, I. V. Grigorieva, and A. A. Firsov, Science 306, 666 (2004).

[11] K. S. Novoselov, A. K. Geim, S. V. Morozov, D. Jiang, M. I. Katsnelson, I. V. Grigorieva, S. V. Dubonos, and A. A. Firsov, Nature 438, 197 (2005).

[12] Y. Zhang, Y.-W. Tan, H. L. Stormer, and P. Kim, Nature 438, 201 (2005).

[13] V. P. Gusynin and S. G. Sharapov, Phys. Rev. Lett. 95, 146801 (2005).

[14] N. M. R. Peres, F. Guinea, and A. H. Castro Neto, Phys. Rev. B 73, 125411 (2006).

[15] H. Kumazaki and D. S. Hirashima, J. Phys. Soc. Jpn. 75, 053707 (2006).

[16] T. Ando, J. Phys. Soc. Jpn. 75, 074716 (2006).

[17] I. F. Herbut, Phys. Rev. Lett. 97, 146401 (2006).

[18] V. M. Pereira, F. Guinea, J. M. B. Lopes dos Santos, N. M. R. Peres, and A. H. Castro Neto, Phys. Rev. Lett. 96, 036801 (2006).

[19] D. V. Khveshchenko, Phys. Rev. Lett. 97, 036802 (2006).

[20] E. McCann, K. Kechedzhi, V. I. Falko, H. Suzuura, T. Ando, and B. L. Altshuler, Phys. Rev. Lett. 97, 146805 (2006).

[21] M. Koshino and T. Ando, Phys. Rev. B 75, 033412 (2007).

[22] Y. Zhang, Z. Jiang, J. P. Small, M. S. Purewal, Y.-W. Tan, M. Fazlollahi, J. D. Chudow, J. A. Jaszczak, H. L. Stormer, and P. Kim, Phys. Rev. Lett. 96, 136806 (2006).

[23] S. V. Morozov, K. S. Novoselov, M. I. Katsnelson, F. Schedin, L. A. Ponomarenko, D. Jiang, and A. K. Geim, Phys. Rev. Lett. 97, 016801 (2006).

[24] M. L. Sadowski, G. Martinez, M. Potemski, noble, C. Berger, and W. A. de Heer, Phys. Rev. Lett. 97, 266405 (2006).

[25] H. Ajiki and T. Ando, J. Phys. Soc. Jpn. 62, 1255 (1993).

[26] T. Ando and T. Nakanishi, J. Phys. Soc. Jpn. 67, 1704 (1998).

[27] T. Ando, T. Nakanishi, and R. Saito, J. Phys. Soc. Jpn. 67, 2857 (1998).

[28] M. V. Berry, Proc. Roy. Soc. London A392, 45 (1984).

[29] J. W. McClure, Phys. Rev. 119, 606 (1960).

[30] M. P. Sharma, L. G. Johnson, and J. W. McClure, Phys. Rev. B 9, 2467 (1974). 
[31] S. A. Safran and F. J. DiSalvo, Phys. Rev. B 20, 4889 (1979).

[32] J. Blinowski and C. Rigaux, J. Phys. (Paris) 45, 545 (1984).

[33] R. Saito and H. Kamimura, Phys. Rev. B 33, 7218 (1986).

[34] E. McCann and V. I. Falko, Phys. Rev. Lett. 96, 086805 (2006)

[35] M. Koshino and T. Ando, Phys. Rev. B 73, 245403 (2006).

[36] S. A. Safran, Phys. Rev. B 30, 421 (1984).

[37] M. Koshino and T. Ando, Phys. Rev. B (submitted for publication).

[38] J. Gonzalez, F. Guinea, and M. A. H. Vozmediano, Nucl. Phys. B 424, 595 (1994).

[39] J. Gonzalez, F. Guinea, and M. A. H. Vozmediano, Phys. Rev. B 59, 2474 (1999).

[40] K. Nomura and A. H. MacDonald, Phys. Rev. Lett. 96, 256602 (2006).

[41] D. P. DiVincenzo and E. J. Mele, Phys. Rev. B 29, 1685 (1984).

[42] F. J. Dyson, J. Math. Phys. 3, 140 (1962).

[43] T. Ando and K. Akimoto, J. Phys. Soc. Jpn. 73, 1895 (2004).

[44] H. Ajiki and T. Ando, J. Phys. Soc. Jpn. 65, 505 (1996).

[45] K. Akimoto and T. Ando, J. Phys. Soc. Jpn. 73, 2194 (2004).

[46] C. L. Kane and E. J. Mele, Phys. Rev. Lett. 78, 1932 (1997).

[47] L. Yang, M. P. Anantram, J. Han, and J. P. Lu, Phys. Rev. B 60, 13874 (1999).

[48] L. Yang and J. Han, Phys. Rev. Lett. 85, 154 (2000).

[49] H. Suzuura and T. Ando, Phys. Rev. B 65, 235412 (2002).

[50] T. Ando, J. Phys. Soc. Jpn. 69, 1757 (2000).

[51] T. Ando and H. Suzuura, J. Phys. Soc. Jpn. 71, 2753 (2002).

[52] H. Ajiki and T. Ando, J. Phys. Soc. Jpn. 62, 2470 (1993); J. Phys. Soc. Jpn. 63, 4267 (1994) (Erratum).

[53] S. Zaric, G. N. Ostojic, J. Kono, J. Shaver, V. C. Moore, M. S. Strano, R. H. Hauge, R. E. Smalley, and X. Wei, Science 304, 1129 (2004).

[54] S. Zaric, G. N. Ostojic, J. Kono, J. Shaver, V. C. Moore, R. H. Hauge, R. E. Smalley, and X. Wei, Nano Lett. 4, 2219 (2004).

[55] S. Zaric, G. N. Ostojic, J. Shaver, J. Kono, O. Portugall, P. H. Frings, G. L. J. A. Rikken, M. Furis, S. A. Crooker, X. Wei, V. C. Moore, R. H. Hauge, and R. E. Smalley, Phys. Rev. Lett. 96, 016406 (2006).
[56] T. Ando, J. Phys. Soc. Jpn. 73, 1273 (2004).

[57] B. L. Al'tshuler, A. G. Aronov, and B. Z. Spivak, Pis'ma Zh. Eksp. Teor. Fiz. 33, 101 (1981) [JETP Lett. 33, 94 (1981)].

[58] D. Yu. Sharvin and Yu. V. Sharvin, Pis'ma Zh. Eksp. Teor. Fiz. 34, 285 (1981) [JETP Lett. 34, 272 (1981)].

[59] T. Ando, J. Phys. Soc. Jpn. 75, 054701 (2006).

[60] H. Suzuura and T. Ando, J. Phys. Soc. Jpn. 75, 024703 (2006).

[61] T. Ando, J. Phys. Soc. Jpn. 71, 2505 (2002).

[62] Y. Asada and T. Ando, J. Phys. Soc. Jpn. 75, 094711 (2006).

[63] M. Igami, T. Nakanishi, and T. Ando, J. Phys. Soc. Jpn. 68, 716 (1999).

[64] M. Igami, T. Nakanishi, and T. Ando, J. Phys. Soc. Jpn. 68, 3146 (1999).

[65] T. Ando, T. Nakanishi, and M. Igami, J. Phys. Soc. Jpn. 68, 3994 (1999).

[66] M. Igami, T. Nakanishi, and T. Ando, J. Phys. Soc. Jpn. 70, 481 (2001).

[67] H. Matsumura and T. Ando, J. Phys. Soc. Jpn. 67, 3542 (1998).

[68] H. Matsumura and T. Ando, J. Phys. Soc. Jpn. 70, 2401 (2001).

[69] H. Matsumura and T. Ando, J. Phys. Soc. Jpn. 70, 2657 (2001).

[70] A. C. Ferrari, J. C. Meyer, V. Scardaci, C. Casiraghi, M. Lazzeri, F. Mauri, S. Piscanec, D. Jiang, K. S. Novoselov, S. Roth, and A. K. Geim, Phys. Rev. Lett. 97, 187401 (2006).

[71] A. Gupta, G. Chena, P. Joshi, S. Tadigadapa, and P. C. Eklund, cond-mat/0606593.

[72] K. Ishikawa and T. Ando, J. Phys. Soc. Jpn. 75, 084713 (2006).

[73] T. Ando, J. Phys. Soc. Jpn. 75, 124701 (2006).

[74] T. Ando, J. Phys. Soc. Jpn. 76, 024712 (2007).

[75] N. A. Viet, H. Ajiki, and T. Ando, J. Phys. Soc. Jpn. 63, 3036 (1994).

[76] M. Lazzeri and F. Mauri, Phys. Rev. B 73, 165419 (2006).

[77] S. Pisana, M. Lazzeri, C. Casiraghi, K. S. Novoselov, A. K. Geim, A. C. Ferrari, F. Mauri, cond-mat/0611714.

[78] J. Yan, Y. Zhang, P. Kim, and Aron Pinczuk, condmat/0612634. 